\title{
Does American Stock Market React Differently to Expected Versus Surprise Ratings During Crisis Period? The Case of the 2008 Worldwide Financial Crisis
}

\author{
Abdelkader Boudriga \\ Associate Professor, Institute of the High Commercial Studies Carthage \\ DEFI Research Unit 4 \\ Rue Abou Zakaria El Hafsi - 1089 Montfleury - Tunis \\ Dorsaf Azouz Ghachem (Corresponding author) \\ ESSEC Tunis, Department of Finance, DEFI Research Unit 4 \\ Rue Abou Zakaria El Hafsi - 1089 Montfleury - Tunis \\ E-mail: dorsafazouz@yahoo.fr
}

Received: July 10, $2018 \quad$ Accepted: August 24, $2018 \quad$ Published: September 2, 2018

doi:10.5296/ijafr.v8i3.13587

URL: https://doi.org/10.5296/ijafr.v8i3.13587

\begin{abstract}
We study the rating impact on American stock market during crisis period by distinguishing expected versus surprise announcements. If unexpected ratings generate stronger reaction than expected ones, which means that rating agencies maintain credibility and influence on investors' decisions. Otherwise, they have to revise their methodologies and procedures in order to recover place on financial markets. Results show that during crisis period market reaction to bad and neutral expected rating announcements is negative and more accentuated than reaction to surprise announcements; on contrary to good news that produce a short positive impact when they are unexpected and are not perceived by the market otherwise. Results reflect once more market distrust to rating agencies and faith loss towards announcements.
\end{abstract}

Keywords: Financial crisis, Expected versus surprise rating, Comparative study event

\section{Introduction}

This paper examines the informational content of rating announcement during crisis periods. Previous studies considered the informational content by examining the impact of rating announcements on stock prices. These studies did not consider the differential impact of 
surprise versus expected announcements.

In one hand, expected information contains data which already have been incorporated by investors and thus may generate reactions prior to official announcement. On the contrary, surprise notations provide new information unknown by investors and thus not yet integrated into stock prices (Dynkin and al., 2002). Therefore, surprise ratings are expected to generate stronger reaction than anticipated ones.

On the other hand, little work is devoted to examine market reaction to rating announcements during crisis periods. Indeed, market prices are expected to react more to ratings during turmoil (Michayluk and Neuhauser, 2006). Furthermore, surprise announcements tend to be less influential on investors decisions since the loss of faith on market information and analysis will lead investors to rely more on personal due diligence than to external assessments (Bahena, 2010). Conversely, we may expect, during crisis, a stronger impact of expected announcements versus surprise ones particularly concerning bad news.

The remainder of the paper is organized as follows. Section 2 reviews some relevant literature on stock market reaction to rating announcements. Section 3 develops the hypotheses. Section 4 presents the methodology and data employed. Section 5 outlines and discusses empirical results. Finally, section 6 concludes the paper.

\section{Market Reaction to Expected Versus Surprise Rating Announcements: Relevant Literature}

The first researches used classical event studies to examine whether market anticipates or not information content of ratings. These are considered to be expected if announcements are preceded by significant abnormal returns. Pinches and Singleton (1978) studied 207 corporate rating announcements by Moody's over a period which extended from 1959 to 1972. They used monthly abnormal returns and concluded that rating changes are expected by investors as they are not followed by significant market reaction.

Griffin and Sanvicente (1982) focused also on monthly abnormal returns of 180 rating changes announced by Moody's and Standard and Poor's over the years 1960 - 1975. They reported an absence of rating expectation. Glascock et al. (1987) based on daily abnormal returns of 162 Moody's rating announcements and concluded to a significant negative reaction surrounding bad announcements.

As regards rating announcements impact on bond prices, the pioneer research of Katz (1973) on monthly abnormal returns of 115 bonds rated by Standard and Poor's during 1966 - 1972, highlighted that market does not anticipate rating announcements. However, three years later, Katz and Grier (1976) studying monthly returns of 96 bonds rated by the same agencies during the same period, concluded that anticipation is conditioned by the firm industry. Industrial values downgrades lead to a strong negative reaction following announcements. Hettenhouse and Sartoris (1976) examined 46 bonds ratings of state-owned companies over the period 1963 to 1973 . They found that market bad news were vaguely expected. Weinstein (1977) studied monthly abnormal returns of 412 bonds rated by Moody's during a period spanning from 1962 to 1974 . He concluded that investors slightly predict rating announcements as no reaction was reported following the announcements. 


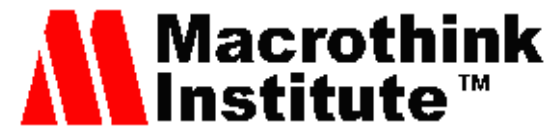

International Journal of Accounting and Financial Reporting

ISSN 2162-3082

2018, Vol. 8, No. 3

Other studies show evidence of asymmetric response to bad versus good expected ratings. Hand et al. (1992) examined 250 rating changes published by Moody's and Standard and Poor's during the period going from 1977 to 1982. They showed that stock prices asymmetrically respond to bad news versus good ones, while bond prices respond similarly to both bad and good rating announcements. Hite and Warga (1997) concentrated on monthly abnormal returns following 1200 rating changes by Moody's and Standard and Poor's extending from 1985 to 1995 . They highlighted that market only expects bad news. Steiner and Heinke (2001) confirmed these results by examining 546 rating changes from the same rating agencies during the same period. Norden and Weber (2004) showed also evidence of asymmetric anticipation and reaction to bad rating news versus good ones.

Di Cesare (2006) argued that abnormal returns prior to rating announcement cannot signal the expected change. He used a Probit model (Note 1) to estimate the probability of a rating occurrence based on significant abnormal returns preceding the rating announcement for CDS spreads and stock prices of 42 banks. He found CDS more effective in anticipating rating occurrence than stock prices. Purda (2007) studied stock prices reaction to expected versus surprise rating news. She concluded that stock market reacts similarly to rating events whether they are expected or not.

Bahena (2010) highlighted that intensive sophistication of financial instruments during the period 2004 to 2006 has made their notation more complex and led to severe shortage in their rating assessments and announcements (both in time and value). Moreover, due to instrument complexity most investors continue to rely on these ratings without referring to their due diligence to assess the instruments' strength. This ultimately led severe market losses and increased suspicion on rating agencies capabilities of timely evaluating firm's financial wealth. Ed DeHaan (2017) showed significant decline in the use of corporate rating after the financial crisis. He highlighted that investors continued to rely more on their own analysis of public information, even during crisis, but less on agencies announcements.

\section{Market Reaction to Expected Versus Surprise Rating Announcements During Crisis Period: Hypothesis Development}

Previous researches offer ambiguous results on the impact of expected rating on stock prices, where significant market reaction is not systematically reported. However, surprise ratings are frequently followed by significant stock prices reaction. Prominently, this might be due to the additional information content that surprise rating news convey.

The majority of existing empirical researches were conducted on a stable context. Reported results can be altered in a crisis period, due to investor's loss of faith in rating agencies and market information treatment ability (Bahena, 2010; DeHaan, 2017).

In this paper, we test the two following hypothesis:

Hypothesis 1: Expected ratings are more accentuated during periods of crisis.

In fact, investors are more interested by firm's news during crisis period, especially concerning bad news (Michayluk and Neuhhauser, 2006). 


\section{MlMacrothink}

International Journal of Accounting and Financial Reporting

ISSN 2162-3082

2018, Vol. 8, No. 3

Hypothesis 2: During crisis episodes, investors react more to bad expected rating announcements in terms of magnitude and duration, but do not respond to good and neutral ones.

In fact, during stable periods, surprise ratings produce stronger effects on stock prices compared to expected ones. Because of increased suspicion, investors tend to lie on their own assessments rather than on publicly available information. Moreover, due to risk aversion, investors are likely to react more intensely to bad news than to good ones (Hand et al., 1992; Hite and Warga, 1997).

\section{Data and Methodology}

\subsection{Data}

Our sample is composed of 216 rating announcements during a crisis period and 135 during a stable period; all diffused from Moody's and Standard and Poor's. Crisis period extends from the 16th September 2008, which coincides with the downturn spread due to the Lehman Brothers bankruptcy, to 31st December 2008. Stable period goes from January 2003 to December 2006 (Note 2). The 2007 year is excluded because it is considered as a noisy year when Subprime crisis emerged and provoked some early investors' reactions.

Announcements which include upgrades, downgrades, assertions and revisions for changes are categorized into three types: good news (upgrades and revisions for upgrade), bad news (downgrades and revisions for downgrade) and neutral news (assertions or affirmations) (Note 3).

Table 1. Descriptive statistics of the crisis and stable periods' samples

\begin{tabular}{lllllll}
\hline & \multicolumn{3}{c}{ Crisis period } & & \multicolumn{3}{c}{ Non crisis period } \\
& Downgrades & Upgrades & Assertions & Downgrades & Upgrades & Assertions \\
\cline { 2 - 7 } Number & 148 & 14 & 54 & 34 & 43 & 58 \\
$\%$ & $68.5 \%$ & $6.5 \%$ & $25 \%$ & $25.19 \%$ & $31.85 \%$ & $42.96 \%$ \\
Total & & & 216 & & & 135 \\
\hline
\end{tabular}

We exclude contaminated observations in order to isolate the impact of ratings on stocks returns. Thus, firms with rating announcements along with other events, such as dividend's distribution or merger announcements are excluded from the sample. We assume that expected rating announcement is preceded by significant cumulative abnormal returns (CAR) (Purda, 2007). We calculate for each announcement the CAR during 120 days (Note 4) before the rating announcement and test for its significance. The announcement is considered as an expected event, if the calculated CAR is significant and as a surprise event otherwise.

Table 2 displays descriptive statistics of expected and unexpected ratings during crisis and stable periods. In both periods, surprise events are more frequent than expected ones. The proportion of both events remains also stable during the two periods (respectively $62.8 \%$ versus $64.71 \%$ and $68.52 \%$ versus $60.34 \%$ ). Conversely, good surprise announcements are more frequent during crisis $(71.43 \%$ versus $58.14 \%)$. 


\section{MlMacrothink}

International Journal of Accounting and Financial Reporting

ISSN 2162-3082

2018, Vol. 8, No. 3

Table 2. Descriptive statistics of expected and non-expected notations during crisis and non-crisis periods

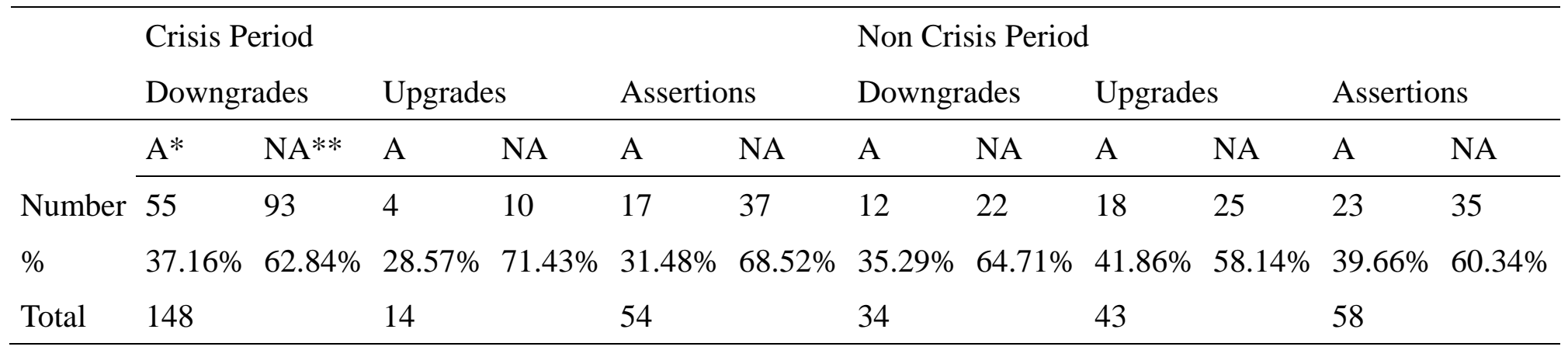

\subsection{Methodology}

\subsubsection{Crisis and Expected Rating Relation}

To identify expected events, we first calculate the daily abnormal returns for the 120 days preceding the rating announcement. Brown and Warner (1985) examined the empirical robustness of several abnormal returns measures. They considered the stock market adjusted return and the market model on daily observations and concluded that both models offer the same power with regard to the theory. The abnormal return (ARi,t) for each event $i$ and each day $t$ is calculated with reference to the stock return adjusted model (1) as the difference between the stock (Rit) and the market (Rmt) returns:

$$
A R_{1 i, t}=R_{i, t}-R_{m, t}
$$

To obtain the cumulative abnormal return (CAR), the daily abnormal returns are summed up over the 120 days as follows:

$$
C A R_{i}=\sum_{t=-120}^{0} A R_{i, t}
$$

Significance test is applied using the $\mathrm{Z}$ test (Note 5). The announcement is considered as an expected event, if the calculated CAR is significant and as a surprise event otherwise.

Events are classified according to two dimensions: the significance of the CARs and the estimation time span. Thus, we obtain four categories of rating announcements: expected ratings during crisis period, surprise ratings during crisis period, expected ratings during stable period and surprise ratings during stable period.

To test the crisis context influence on rating expectation on the level of CARs, we apply the independence Chi Squared test. Crisis is a binary variable that equals 1 for the crisis window and 0 otherwise. CAR is also a binary variable that equals 1 if the cumulative abnormal return preceding the announcement is significant and 0 otherwise. For each type of observations, we calculate the Chi squared test. 


\section{Mll Macrothink}

International Journal of Accounting and Financial Reporting

ISSN 2162-3082

2018, Vol. 8, No. 3

4.2.2 Impact of Rating Nature (Expected versus Surprise) on Stock Market Prices During Crisis Period

We use the event study methodology to assess the rating impact on stock prices. The event window extends over 20 days symmetrically around the rating announcement day.

We first calculate abnormal returns for each type of event, using two models: the stock index adjusted model (1) and the market return adjusted model (2). The latter computes the difference between observed and theoretical returns using the market model. The estimation period covers 255 days before the first day of the event window (day -10).

$$
A R_{2 i, t}=R_{i, t}-\alpha_{i}-\beta_{i} * R_{m, t}
$$

Where $\alpha_{\mathrm{i}}$ and $\beta_{\mathrm{i}}$ are the market model estimated values.

Then, we calculate the mean abnormal and the mean cumulative abnormal daily returns. Finally, we compare results for two periods with regard to time persistence and magnitude.

\section{Empirical Results}

\subsection{Crisis and Rating Expectation}

We find a significant correlation between crisis context and expected ratings for both of bad and good rating news. By contrast, neutral expected announcements are not accentuated during crisis period. This result shows that investors give careful consideration to bad and good rating news during crisis period. This shall not apply to neutral ratings owing to the weak informational input they contain.

Consequently, we accept the first hypothesis: expected bad and good ratings are more pronounced during crisis period.

Table 3. Chi Squared Independence test between crisis and expected ratings

\begin{tabular}{llll}
\hline & Downgrades & Upgrades & Assertions \\
\cline { 2 - 4 }$\chi^{2}$ & $71.4^{* * *}$ & $14.7^{* * * *}$ & 0.143 \\
p-value & 0.000 & 0.000 & 0.705 \\
\hline
\end{tabular}

\subsection{Impact of Expected Versus Surprise Ratings During Crisis Period}

\subsubsection{Impact of Expected Versus Surprise Bad Ratings During Crisis Period}

Results of ARs and CARs following bad news during crisis period are respectively presented in Figures 1 and 2 below: 


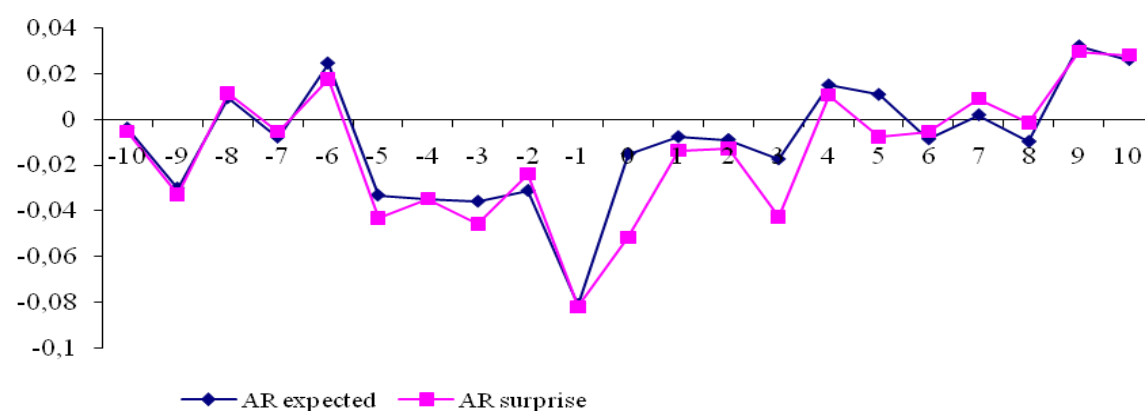

Figure 1. ARs following bad rating events during crisis

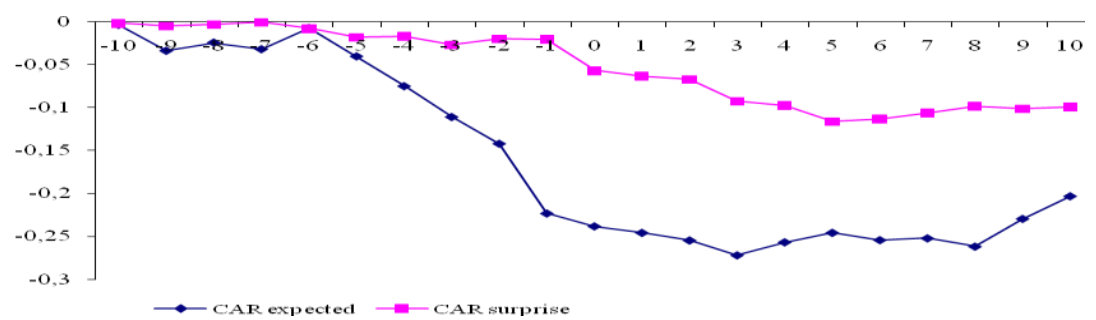

Figure 2. CARs following bad rating events during crisis

As regards expected ratings, significant negative ARs and CARs are observed before the event day. ARs extend from five days $(-3.31 \%)$ to one day $(-8.1 \%)$ before the announcement and become non-significant after. Significant negative CARs are observed from three days $(-11 \%)$ before the announcement to nine days after (-22.9\%). Conversely, surprise ratings are not preceded by significant ARs and CARs; these become slightly negative after the announcement (Note 6).

Based on the Figure 2, CARs following expected rating news are stronger and more persistent than those following surprise announcements during crisis period. Expected ratings have bigger impact on stock prices in terms of magnitude and duration.

Figures 3 and 4 display respectively ARs and CARs following expected and surprise bad rating news during the stable period. Once more, expected bad rating news have stronger impact on stock prices in terms of time and magnitude.

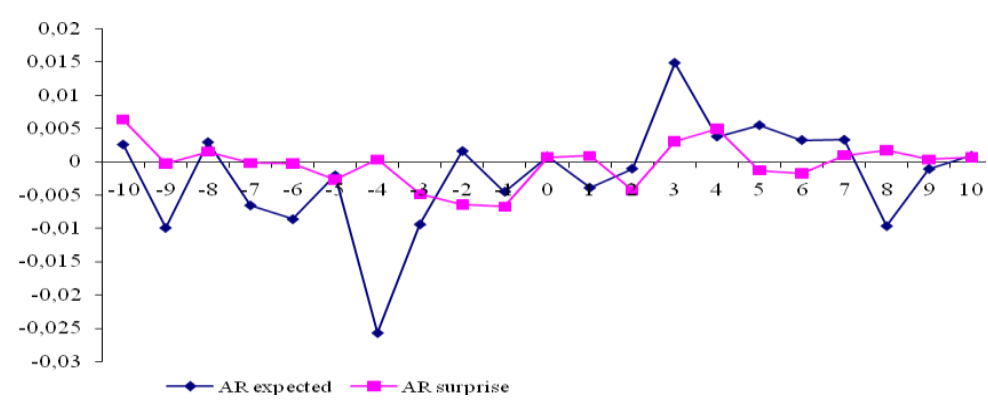

Figure 3. ARs following bad rating events during non-crisis 


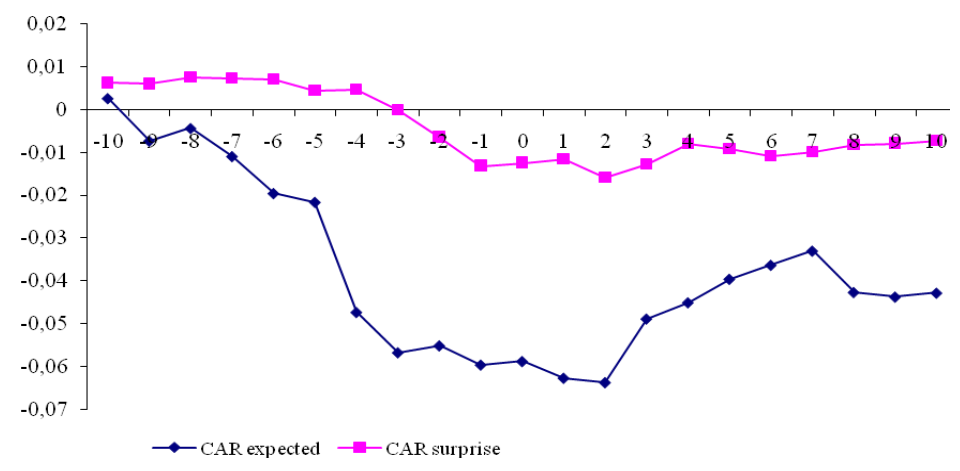

Figure 4. CARs following bad rating events during non-crisis

ARs following expected announcements are not significant. Reversely, CARs are significant from day $-4(-4.7 \%)$ to day $+3(-4.9 \%)$. However, surprise bad rating announcements have no significant impact on prices.

These results highlight two main conclusions. Firstly, during crisis period, investors overreact to both of expected and surprise bad ratings (Glascock et al., 1987; Norden and Weber, 2004). Secondly, expected announcements have stronger impact on stock prices in terms of magnitude and duration. Our results might be explained by the impact of public information on investors 'decisions (DeHaan, 2017).

To examine the robustness of our results, we apply mean difference tests to ARs and CARs with regard to ratings' types (expected versus surprise) and periods (crisis versus stability). The null hypothesis states that mean differences are null.

Table 4a displays ARs and CARs mean differences by reference to ratings' types. ARs mean differences are only significant for the day $-2(-3,8 \%)$ and day $-1(-8 \%)$. Significant CARs mean differences appear 2 days before the announcement $(-12,2 \%)$ and persist over 4 days $(-15,9 \%)$. We therefore conclude that, during crisis period, expected bad rating events exhibit more pronounced impact on stock prices in terms of magnitude and duration.

Table 4a. Mean difference tests between ARs and CARs following expected versus surprise bad news during crisis period

\begin{tabular}{|c|c|c|c|c|c|c|c|c|}
\hline \multirow[b]{2}{*}{ Day $(t)$} & \multicolumn{4}{|c|}{ Stock Index Adjusted Model } & \multicolumn{4}{|c|}{ Market Return Adjusted Model } \\
\hline & $\mathrm{AR}$ & $\mathrm{t}$-stat & CAR & $\mathrm{t}$-stat & $\mathrm{AR}$ & $\mathrm{t}$-stat & CAR & $\mathrm{t}$-stat \\
\hline-10 & -0.00208 & -0.097 & -0.00208 & -0.097 & -0.00696 & -0.324 & -0.00696 & -0.324 \\
\hline-9 & -0.02729 & -1.616 & -0.02937 & -0.939 & -0.02870 & -1.645 & -0.03566 & -1.096 \\
\hline-8 & 0.00774 & 0.427 & -0.02163 & -0.615 & 0.01015 & 0.568 & -0.02550 & -0.698 \\
\hline-7 & -0.00995 & -0.517 & -0.03158 & -0.853 & -0.02057 & -1.048 & -0.04608 & -1.124 \\
\hline-6 & 0.03213 & 0.938 & 0.00054 & 0.012 & 0.03151 & 0.943 & -0.01457 & -0.297 \\
\hline-5 & -0.02309 & -1.446 & -0.02254 & -0.492 & -0.02096 & -1.295 & -0.02263 & -0.488 \\
\hline-4 & $-0.03586^{*}$ & -1.851 & -0.05840 & -1.236 & -0.02830 & -1.483 & -0.05093 & -1.051 \\
\hline
\end{tabular}




\begin{tabular}{|c|c|c|c|c|c|c|c|c|}
\hline \multirow[b]{2}{*}{-3} & \multirow[b]{2}{*}{-0.02593} & \multirow[b]{2}{*}{-1.649} & \multicolumn{2}{|c|}{ International $\mathbf{J}$} & \multicolumn{2}{|c|}{ Journal of Accounting and } & \multicolumn{2}{|c|}{$\begin{array}{r}\text { Financial Reporting } \\
\text { ISSN 2162-3082 } \\
\text { 2018, Vol. 8, No. } 3 \\
\end{array}$} \\
\hline & & & $-0.08434 *$ & -1.727 & $-0.03275^{*}$ & -1.955 & -0.08369 & -1.639 \\
\hline-2 & $-0.03829 * *$ & -2.065 & $-0.12263 * *$ & -2.402 & $-0.03735^{* *}$ & -2.007 & $-0.12104 * *$ & -2.306 \\
\hline-1 & $-0.08028 * * *$ & -3.638 & $-0.20292 * * *$ & -4.026 & $-0.07451 * * *$ & -3.386 & $-0.19555 * * *$ & -3.741 \\
\hline 0 & 0.02162 & 0.708 & $-0.18129 * * *$ & -3.261 & 0.02762 & 0.912 & $-0.16793 * * *$ & -2.958 \\
\hline 1 & -0.00131 & -0.006 & $-0.18260 * * *$ & -3.077 & -0.00200 & -0.090 & $-0.16993 * * *$ & -2.770 \\
\hline 2 & -0.00476 & -0.288 & $-0.18737 * * *$ & -2.855 & -0.00895 & -0.514 & $-0.17888 * *$ & -2.609 \\
\hline 3 & 0.00804 & 0.456 & $-0.17933^{* *}$ & -2.637 & 0.01141 & 0.653 & $-0.16746^{* *}$ & -2.372 \\
\hline 4 & 0.01993 & 1.160 & $-0.15939 * *$ & -2.438 & 0.02477 & 1.462 & $-0.14269 * *$ & -2.057 \\
\hline 5 & 0.02962 & 1.400 & -0.12976 & -1.797 & 0.02664 & 1.258 & -0.11604 & -1.500 \\
\hline 6 & -0.01153 & -0.639 & $-0.14130^{*}$ & -1.908 & 0.00193 & 0.095 & -0.11411 & -1.488 \\
\hline 7 & -0.00473 & -0.199 & $-0.14604^{*}$ & -1.804 & -0.00256 & -0.108 & -0.11667 & -1.393 \\
\hline 8 & -0.01756 & -0.846 & $-0.16360 * *$ & -2.017 & -0.01541 & -0.764 & -0.13209 & -1.576 \\
\hline 9 & 0.03513 & 1.291 & -0.12846 & -1.614 & 0.03893 & 1.476 & -0.09315 & -1.120 \\
\hline 10 & 0.02448 & 0.992 & -0.10398 & -1.282 & 0.02154 & 0.880 & -0.07161 & -0.840 \\
\hline
\end{tabular}

$* * *, * *$ and $*$ denote respectively significance at $1 \%, 5 \%$ and $10 \%$ levels.

Next, we examine results for the stable period. Table $4 \mathrm{~b}$ displays ARs and CARs mean differences between expected and surprise events. ARs mean differences are only significant before the announcement, i.e. during day $-4(-2,6 \%)$ and day $-2(-0,8 \%)$. Conversely, no significant CARs mean differences are reported for the calm period. Thus, it seems that stock market reacts similarly to both types of ratings during the normal period (Purda, 2007).

Table 4b. Mean difference tests between ARs and CARs following expected versus surprise bad news during stable period

\begin{tabular}{|c|c|c|c|c|c|c|c|c|}
\hline & \multicolumn{4}{|c|}{ Stock Index Adjusted Model } & \multicolumn{4}{|c|}{ Market Return Adjusted Model } \\
\hline Day $(t)$ & AR & $\mathrm{t}$-stat & CAR & $\mathrm{t}$-stat & AR & $\mathrm{t}$-stat & CAR & $\mathrm{t}$-stat \\
\hline-10 & -0.00368 & -0.413 & -0.00368 & -0.413 & -0.00277 & -0.310 & -0.00277 & -0.310 \\
\hline-9 & -0.00968 & -1.031 & -0.01337 & -1.392 & -0.00799 & -0.809 & -0.01077 & -1.009 \\
\hline-8 & 0.00142 & 0.104 & -0.01195 & -0.838 & 0.00342 & 0.247 & -0.00734 & -0.418 \\
\hline-7 & -0.00638 & -1.036 & -0.01833 & -1.178 & -0.00573 & -0.510 & -0.01308 & -0.830 \\
\hline-6 & -0.00839 & -1.332 & -0.02672 & -1.624 & -0.00588 & -0.942 & -0.01897 & -1.165 \\
\hline-5 & 0.00061 & 0.097 & -0.02611 & -1.331 & 0.00293 & 0.467 & -0.01603 & -0.837 \\
\hline-4 & $-0.02610 *$ & -1.834 & $-0.05221 *$ & -2.118 & $-0.02463^{*}$ & -1.753 & -0.04066 & -1.679 \\
\hline-3 & -0.00458 & -0.334 & $-0.05680 *$ & -2.068 & -0.00357 & -0.264 & -0.04423 & -1.656 \\
\hline-2 & $0.00799 *$ & 1.707 & $-0.04881^{*}$ & -1.823 & $0.00890 *$ & 1.857 & -0.03533 & -1.348 \\
\hline
\end{tabular}




\section{MlMacrothink}

International Journal of Accounting and Financial Reporting ISSN 2162-3082 2018, Vol. 8, No. 3

\begin{tabular}{lllllllll}
-1 & 0.00219 & 0.373 & $-0.04661^{*}$ & -1.788 & 0.00509 & 0.908 & -0.03024 & -1.183 \\
0 & 0.00010 & 0.013 & -0.04651 & -1.702 & 0.00333 & 0.451 & -0.02691 & -1.006 \\
1 & -0.00478 & -0.757 & -0.05129 & -1.765 & -0.00466 & -0.684 & -0.03157 & -1.108 \\
2 & 0.00329 & 0.341 & $-0.04800^{*}$ & -1.741 & 0.00474 & 0.516 & -0.02683 & -0.991 \\
3 & 0.01185 & 0.892 & -0.03615 & -1.482 & 0.01307 & 0.955 & -0.01375 & -0.549 \\
4 & -0.00110 & -0.123 & -0.03725 & -1.429 & -0.00112 & -0.130 & -0.01487 & -0.543 \\
5 & 0.00680 & 1.087 & -0.03044 & -1.140 & 0.00787 & 1.277 & -0.00699 & -0.252 \\
6 & 0.00498 & 0.963 & -0.02546 & -0.924 & 0.00528 & 0.947 & -0.00171 & -0.059 \\
7 & 0.00234 & 0.406 & -0.02312 & -0.829 & 0.00456 & 0.785 & 0.00285 & 0.097 \\
8 & $-0.01141 *$ & -1.724 & -0.03453 & -1.223 & -0.00828 & -1.291 & -0.00543 & -0.184 \\
9 & -0.00143 & -0.231 & -0.03596 & -1.128 & -0.00138 & -0.219 & -0.00682 & -0.205 \\
10 & 0.00028 & 0.032 & -0.03568 & -1.192 & 0.00239 & 0.284 & -0.00442 & -0.140 \\
\hline
\end{tabular}

$* * *, * *$ and $*$ denote respectively significance at $1 \%, 5 \%$ and $10 \%$ levels.

Globally, results confirm the overreaction hypothesis to bad ratings during crisis for both of the expected and the surprise ratings. However, expected announcements have stronger impact on stock prices. This might be due to investor's loss of faith in rating agencies during turmoils (Michayluk and Neuhauser, 2006).

In fact, investors are akin to trust expected rating announcements, which were seriously and thoroughly analyzed. Expected ratings are then followed by important prices drops due to accumulated and considerable selling by investors.

Conversely, surprise bad rating announcements generate weaker negative impact on prices in terms of magnitude and duration. This confirms once again the deterioration of confidence in rating agencies. Investors react less to surprise announcements, which are transmitted only by rating agencies. Finally, we study the impact of the crisis on expected and surprise bad rating events.

Table 5a. Mean difference tests between ARs and CARs following expected bad news during crisis versus stable period

\begin{tabular}{|c|c|c|c|c|c|c|c|c|}
\hline \multirow[b]{2}{*}{ Day $(\mathrm{t})$} & \multicolumn{3}{|c|}{ Stock Index Adjusted Model } & \multicolumn{4}{|c|}{ Market Return Adjusted Model } & \multirow[b]{2}{*}{$\mathrm{t}$-stat } \\
\hline & $\mathrm{AR}$ & $\mathrm{t}$-stat & CAR & $\mathrm{t}$-stat & & AR $\mathrm{t}$-sta & at CAR & \\
\hline-10 & -0.00637 & -0.289 & -0.00637 & -0.289 & -0.00652 & $-0.303-0$ & 0.00652 & -0.303 \\
\hline-9 & -0.02014 & -1.106 & -0.02651 & -0.852 & -0.01676 & $-0.913-0$ & 0.02328 & -0.746 \\
\hline-8 & 0.00655 & 0.310 & -0.01996 & -0.552 & 0.01151 & $0.542-0$ & 0.01176 & -0.326 \\
\hline-7 & -0.00110 & -0.059 & -0.02107 & -0.553 & -0.00347 & $-0.196 \quad .0$ & 01523 & -0.401 \\
\hline-6 & 0.03340 & 0.987 & 0.01233 & 0.275 & 0.03426 & 1.0370 & 0.01903 & 0.426 \\
\hline-5 & $-0.03105^{* *}$ & -2.159 & -0.01872 & -0.403 & $-0.03144 * *$ & $-2.152-0$ & 0.01241 & -0.270 \\
\hline
\end{tabular}




\begin{tabular}{ccccccccc}
-4 & -0.00886 & -0.393 & -0.02759 & -0.554 & -0.00454 & -0.205 & -0.01695 & -0.343 \\
-3 & -0.02641 & -1.348 & -0.05400 & -1.032 & -0.02092 & -1.087 & -0.03788 & -0.740 \\
-2 & $-0.03273 *$ & -1.933 & -0.08673 & -1.606 & $-0.03422^{* *}$ & -2.054 & -0.07210 & -1.356 \\
-1 & $-0.07657 * * *$ & -3.535 & $-0.16330^{* * *}$ & -3.084 & $-0.07274 * * *$ & -3.371 & $-0.1448 * * *$ & -2.758 \\
0 & -0.01583 & -0.551 & $-0.17913 * * *$ & -3.152 & -0.01365 & -0.490 & $-0.15850 * * *$ & -2.797 \\
1 & -0.00362 & -0.194 & $-0.18276^{* * *}$ & -2.997 & -0.00395 & -0.220 & $-0.16245^{* * * *}$ & -2.664 \\
2 & -0.00778 & -0.489 & $-0.19055^{* * *}$ & -2.928 & -0.00362 & -0.235 & $-0.16608 * *$ & -2.560 \\
3 & $-0.03221 *$ & -1.602 & $-0.22276^{* * *}$ & -3.387 & -0.02621 & -1.316 & $-0.19229 * * *$ & -2.925 \\
4 & 0.01137 & 0.656 & $-0.21138^{* * *}$ & -3.344 & 0.01891 & 1.125 & $-0.17338 * * *$ & -2.724 \\
5 & 0.00548 & 0.267 & $-0.20590^{* * *}$ & -2.987 & 0.00833 & 0.426 & $-0.16504 * *$ & -2.388 \\
6 & -0.01174 & -0.671 & $-0.21765^{* * *}$ & -3.038 & -0.00940 & -0.540 & $-0.17445 * *$ & -2.411 \\
7 & -0.00123 & -0.054 & $-0.21889^{* * *}$ & -2.793 & 0.00246 & 0.109 & $-0.17199 * *$ & -2.163 \\
8 & 0.00005 & 0.003 & $-0.21884 * * *$ & -2.773 & -0.00113 & -0.075 & $-0.17312 * *$ & -2.165 \\
9 & 0.03334 & 1.291 & $-0.18549 * *$ & -2.369 & 0.03534 & 1.416 & $-0.13778 *$ & -1.716 \\
10 & 0.02537 & 1.023 & $-0.16012^{* * *}$ & -2.034 & 0.02520 & 1.030 & -0.11258 & -1.385 \\
\hline
\end{tabular}

$* * *, * *$ and $*$ denote respectively significance at $1 \%, 5 \%$ and $10 \%$ levels.

Table 5b. Mean difference tests between ARs and CARs following surprise bad news during crisis versus stable period

\begin{tabular}{|c|c|c|c|c|c|c|c|c|}
\hline \multicolumn{5}{|c|}{ Stock Index Adjusted Model } & \multicolumn{4}{|c|}{ Market Return Adjusted Model } \\
\hline $\operatorname{Day}(\mathrm{t})$ & t) $\mathrm{AR}$ & $\mathrm{t}$-stat & CAR & $\mathrm{t}$-stat & AR & $\mathrm{t}$-stat & CAR & $\mathrm{t}$-stat \\
\hline$-10-$ & -0.00797 & -1.073 & -0.00797 & -1.073 & -0.00233 & -0.265 & -0.00233 & -0.265 \\
\hline-9 & -0.00253 & -0.389 & -0.01050 & -1.036 & 0.00394 & 0.487 & 0.00161 & 0.114 \\
\hline-8 & 0.00022 & 0.027 & -0.01028 & -0.884 & 0.00478 & 0.618 & 0.00639 & 0.413 \\
\hline-7 & 0.00246 & 0.305 & -0.00781 & -0.612 & 0.01136 & 1.060 & 0.01776 & 0.806 \\
\hline-6 & -0.00712 & -0.869 & -0.01494 & -0.977 & -0.00313 & -0.391 & 0.01463 & 0561 \\
\hline-5 & -0.00734 & -0.784 & -0.02228 & -1.237 & -0.02044 & -1.330 & -0.00581 & -0.288 \\
\hline-4 & 0.00088 & 0.107 & -0.02140 & -1.121 & -0.00086 & -0.102 & -0.00668 & -0.300 \\
\hline-3 & -0.00506 & -0.701 & -0.02646 & -1.320 & 0.00824 & 0.859 & 0.00156 & 0.059 \\
\hline-2 & 0.01355 & 1.524 & -0.01290 & -0.639 & 0.01203 & 1.258 & 0.01360 & 0.549 \\
\hline-1 & 0.00590 & 0.816 & -0.00700 & -0.343 & 0.00685 & 0.970 & 0.02046 & 0.816 \\
\hline 0 & $-0.03735 * * *$ & -2.902 & $-0.04435^{*}$ & -1.799 & $-0.03794 * * *$ & -2.709 & -0.01748 & -0.649 \\
\hline 1 & -0.00709 & -0.542 & $-0.05144 * *$ & -2.023 & -0.00661 & -0.453 & -0.02409 & -0.823 \\
\hline
\end{tabular}




\begin{tabular}{|c|c|c|c|c|c|c|c|}
\hline 0.00026 & 0.025 & $-0.05118^{*}$ & -1.773 & 0.01006 & 0.824 & -0.01402 & -0.4 \\
\hline$-0.02839 * * *$ & -3.112 & $-0.07958 * * *$ & -2.664 & $-0.02455^{* *}$ & -2.501 & -0.03858 & -1.073 \\
\hline-0.00967 & -1.115 & $-0.08925 * * *$ & -2.887 & -0.00698 & -0.791 & -0.04556 & -1.171 \\
\hline$-0.01733 * *$ & -2.136 & $-0.10658 * * *$ & -3.107 & -0.01042 & -1.021 & -0.05599 & -1.259 \\
\hline 0.00476 & 0.703 & $-0.10181 * * *$ & -3.059 & -0.00605 & -0.515 & -0.06205 & -1.612 \\
\hline 0.00584 & 0.613 & $-0.09597 * * *$ & -2.784 & 0.00958 & 1.057 & -0.05246 & -1.332 \\
\hline 0.00619 & 0.425 & $-0.08977 * * *$ & -2.646 & 0.00599 & 0.405 & -0.04647 & -1.198 \\
\hline-0.00322 & -0.304 & $-0.09299 * * *$ & -2.662 & -0.00497 & -0.470 & -0.05145 & -1.297 \\
\hline 0.00116 & 0.142 & $-0.09183^{* *}$ & -2.576 & 0.00605 & 0.714 & -0.04539 & -1.112 \\
\hline
\end{tabular}

$* * *, * *$ and $*$ denote respectively significance at $1 \%, 5 \%$ and $10 \%$ levels.

Table 5a displays ARs and CARs mean differences between crisis and stable periods of the expected bad news. The null hypothesis states that mean differences are null. ARs significant differences appear in day $-5(-3,1 \%)$, day $-2(-3,2 \%)$ and day $-1(-7,6 \%)$. CARs mean differences extend from day $-1(-16,3 \%)$ to day $+9(-18.5 \%)$. We conclude that crisis amplifies the impact of expected bad rating news on stock prices.

Table 5b displays ARs and CARs mean difference tests of surprise bad news between crisis and normal periods. We find similar results as for expected news. It appears also that crisis amplifies market reaction to surprise downgrades. Mean differences are significant in day 0 $(-3,7 \%)$, day $+3(-2.8 \%)$ and day $+5(-1,7 \%)$ for ARs, and from day $+1(-5,1 \%)$ to day +10 $(-9,1 \%)$ for CARs.

Globally, we retain that investors overreact to bad rating news during crisis period, whether announcements are expected or not. However, reaction to expected downgrades is stronger, which is not valid in stable periods. Also, crisis amplifies market reaction for both of the expected and the surprise bad announcements. The market behavior is explained by risk aversion in one hand, and loss of faith in rating agencies, in the other hand. In fact, investors feel trustful of expected bad ratings they thought carefully, and react less to surprise announcements.

\subsubsection{Impact of Expected Versus Surprise Good Ratings During Crisis Period}

Figures 5 and 6 present ARs and CARs following expected and surprise upgrades during the crisis period (Note 7). They reveal that good ratings do not have impact on stock prices, whether they are expected or not. Asymmetric reaction to bad versus good news is proved once again. The results confirm the investor's carefulness towards good news during downturns. In fact, the absence of impact of good news is explained by the asymmetric informational role of rating agencies. Firms tend to report quickly good news to which investors react before the rating announcement (Griffin and Sanvicente, 1982). Consequently, good news have no significant impact on stock prices after the dissemination of the upgrade. Conversely, bad news are solely reported by rating agencies, which produces a substantial negative impact on stock 


\section{MlMacrothink}

International Journal of Accounting and Financial Reporting

ISSN 2162-3082

2018, Vol. 8, No. 3

prices (Ederington and Goh, 1993). This asymmetric impact of bad versus good ratings is more pronounced for expected announcements.

On the contrary, surprise good rating announcements have a very short and positive impact; CARs are significantly positive only the day $0(+13.1 \%)$ and day $+1(+13.8 \%)$.

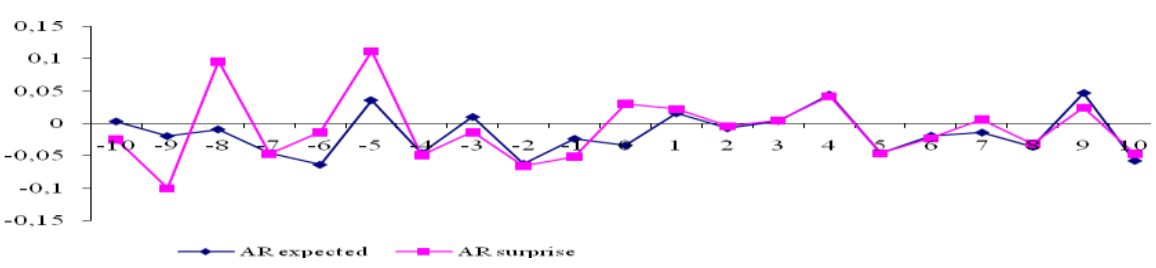

Figure 5. ARs following good rating events during crisis

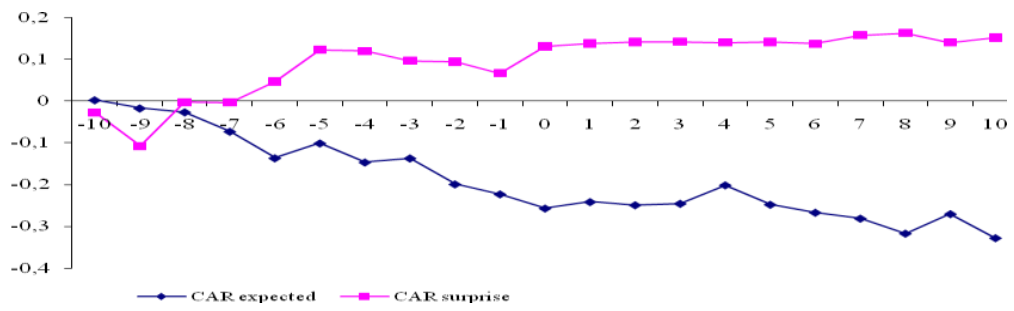

Figure 6. CARs following good rating events during crisis

Finally, we note that results corroborate our second hypothesis. Expected and surprise good ratings have no persistent effect on stock prices during financial downturns. In fact, expected upgrades do not impact stock prices; unlike surprise ones which have a very short and positive effect on prices. It should be noted also that investors are aware of conflicts of interests between credit rating agencies and issued firms because of the existence of paid fees. Moreover, rating inflation is assumed to increase agencies revenues. Also, it seems that upgrades are motivated by institutional investors' pressures as they are required to hold high quality assets. Finally, opaque issuers require more ratings and present a great activity potential for rating agencies which tend to inflate their cotes (Cornaggia et al., 2017).

These results turn to be different during stable period. Figures 7 and 8 show respectively ARs and CARs following expected and surprise good rating news in a normal context. 


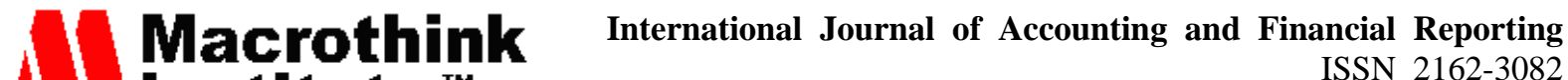

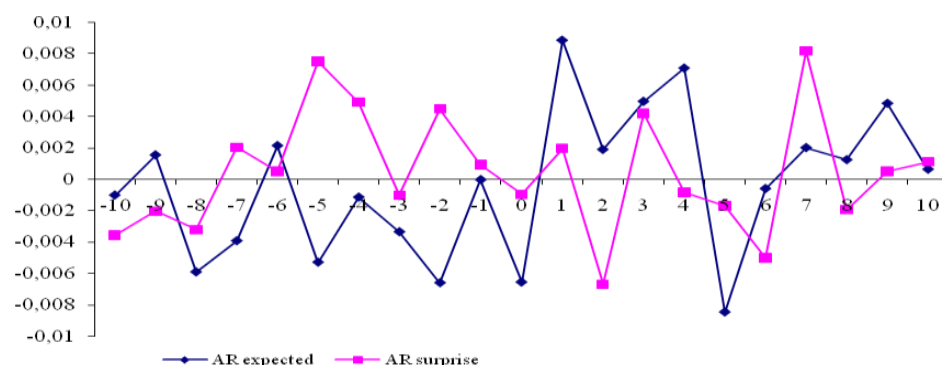

Figure 7. ARs following good rating events during non-crisis

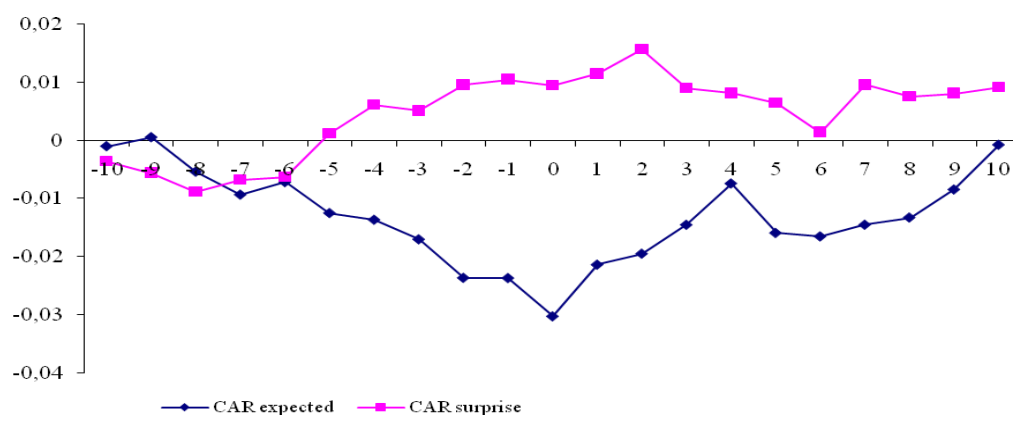

Figure 8. CARs following good rating events during non-crisis

Expected good rating news generate significant ARs in day $-2(-0.6 \%)$, day $+1(0.8 \%)$ and day $+5(-0.8 \%)$. On the contrary, unexpected positive rating events are favorably incorporated three days after the announcement, with an abnormal return equal to $+0.4 \%$. However, CARs are not significant neither for expected nor for surprise good news. This result points out that upgrades and revisions for upgrades have no effect on stock prices during the stable period. Once again, it highlights the short and weak impact of good rating news on stock markets (Gallo and Velluchi, 2009).

To check the robustness of our results, we apply mean difference tests for ARs and CARs for each type of upgrades (expected versus surprise).

Starting with the crisis period, table 6a displays mean difference tests of expected and surprise ARs and CARs. Results show that ARs differences are not significant around the announcement day, whereas significant CARs mean differences extend from the day $-5(-22$, $4 \%)$ to day $+10(-48,1 \%)$.

However, it is interesting to note that absolute values of CARs following expected good ratings are higher than those of surprise ones during crisis period. Differences are explained by a different reaction to expected versus surprise announcements during downturns. In fact, expected upgrades provoke negative reaction, while surprise upgrades exhibit a very short and positive impact on stock prices.

The unfavorable reaction to expected upgrades might be explained by the possible wealth transfer from stockholders to bond creditors revealed by those announcements. Besides, the repayment priority is transferred to bond creditors before stockholders, especially during 
turmoil. Consequently, expected upgrades are not considered as good news for stockholders. This adverse reaction is stronger during crisis period.

Table $6 \mathrm{~b}$ displays ARs and CARs mean difference tests following expected versus surprise upgrades during the normal period. AR mean differences are significant during the day $-5(-1$, $2 \%)$ and day $+3(1,1 \%)$; contrary to CAR mean differences which are insignificant. These results prove once again the similarity of reaction to both expected and surprise ratings (Purda, 2007).

Table 6a. Mean difference tests between ARs and CARs following expected versus surprise good news during crisis period

\begin{tabular}{|c|c|c|c|c|c|c|c|c|}
\hline \multicolumn{4}{|c|}{ Stock Index Adjusted Model } & \multicolumn{5}{|c|}{ Market Return Adjusted Model } \\
\hline $\operatorname{Day}(\mathrm{t})$ & $\mathrm{AR}$ & $\mathrm{t}$-stat & CAR & $\mathrm{t}$-stat & AR & $\mathrm{t}$-stat & CAR & $\mathrm{t}$-stat \\
\hline-10 & 0.03040 & 0.516 & 0.03040 & 0.516 & 0.03100 & 0.547 & 0.03100 & 0.547 \\
\hline-9 & 0.06053 & 0.409 & 0.09094 & 0.717 & 0.04930 & 0.452 & 0.08030 & 0.772 \\
\hline-8 & -0.11428 & -0.669 & -0.02334 & -0.393 & -0.10521 & -0.692 & 0.02490 & 0.458 \\
\hline-7 & $-0.04635 * * *$ & -3.177 & -0.06970 & -1.278 & $-0.04393 * *$ & -2.306 & -0.06883 & -1.099 \\
\hline-6 & $-0.11431 * * *$ & -3.272 & -0.18401 & -2.393 & $-0.11956^{* *}$ & -2.305 & -0.18840 & -2.375 \\
\hline-5 & -0.04026 & -0.334 & $-0.22428 *$ & -2.181 & -0.04104 & -0.329 & $-0.22944 *$ & -1.895 \\
\hline-4 & -0.04287 & -1.335 & $-0.26716 * * *$ & -3.598 & -0.05383 & -1.512 & $-0.28327 * *$ & -2.606 \\
\hline-3 & 0.03302 & 0.760 & $-0.23413 * * *$ & -3.810 & 0.01980 & 0.493 & $-0.26347 * *$ & -2.352 \\
\hline-2 & $-0.05946 * *$ & -2.302 & $-0.29360 * * *$ & -3.911 & -0.04072 & -1.611 & $-0.30420 * *$ & -2.427 \\
\hline-1 & 0.00335 & 0.074 & $-0.29024 * * *$ & -4.252 & -0.01285 & -0.741 & $-0.31706^{* *}$ & -2.417 \\
\hline 0 & -0.09874 & -1.111 & $-0.38899 * *$ & -3.367 & -0.09323 & -0.974 & $-0.41029 *$ & -2.214 \\
\hline 1 & 0.00872 & 0.290 & $-0.38027 * *$ & -3.228 & 0.04569 & 0.811 & $-0.36460 *$ & -2.232 \\
\hline 2 & -0.01121 & -0.337 & $-0.39148 * * *$ & -3.718 & -0.01750 & -0.589 & $-0.38211^{* *}$ & -2.374 \\
\hline 3 & 0.00251 & 0.077 & $-0.38897 * * *$ & -3.381 & -0.03684 & -0.987 & $-0.41895^{*}$ & -2.203 \\
\hline 4 & 0.04644 & 1.695 & $-0.34252 * *$ & -2.694 & $0.06618 * *$ & 2.658 & $-0.35277 *$ & -1.856 \\
\hline 5 & $-0.04750^{*}$ & -1.830 & $-0.39003 * * *$ & -3.287 & -0.02444 & -1.006 & $-0.37722^{*}$ & -2.106 \\
\hline 6 & -0.01529 & -0.898 & $-0.40532 * *$ & -3.324 & -0.00962 & -0.279 & $-0.38684 * *$ & -2.299 \\
\hline 7 & -0.03482 & -1.274 & $-0.44015 * * *$ & -3.631 & -0.03229 & -0.872 & $-0.41940 * *$ & -2.295 \\
\hline 8 & -0.04098 & -1.590 & $-0.48113 * * *$ & -3.581 & -0.04583 & -1.236 & $-0.46497 * *$ & -2.276 \\
\hline 9 & $0.06891 * * *$ & 3.644 & $-0.41222 * *$ & -3.182 & $0.06176^{* *}$ & 2.550 & $-0.40321 *$ & -1.953 \\
\hline 10 & $-0.06890 * *$ & -2.811 & $-0.48112 * * *$ & -3.523 & -0.06106 & -1.754 & $-0.46427 *$ & -2.044 \\
\hline
\end{tabular}

$* * *, * *$ and $*$ denote respectively significance at $1 \%, 5 \%$ and $10 \%$ levels. 
Table 6b. Mean difference tests between ARs and CARs following expected versus surprise good news during stable period

\begin{tabular}{|c|c|c|c|c|c|c|c|c|}
\hline & \multicolumn{4}{|c|}{ Stock Index Adjusted Model } & \multicolumn{4}{|c|}{ Market Return Adjusted Model } \\
\hline Day $(t)$ & $\mathrm{AR}$ & $\mathrm{t}$-stat & CAR & $\mathrm{t}$-stat & AR & $\mathrm{t}$-stat & CAR & $\mathrm{t}$-stat \\
\hline-10 & 0.00254 & 0.534 & 0.00254 & 0.534 & 0.00523 & 1.177 & 0.00523 & 1.177 \\
\hline-9 & 0.00358 & 0.845 & 0.00613 & 0.894 & $0.00618^{*}$ & 1.524 & $0.01141 *$ & 1.912 \\
\hline-8 & -0.00270 & -0.499 & 0.00342 & 0.354 & -0.00181 & -0.309 & 0.00960 & 1.038 \\
\hline-7 & -0.00596 & -0.790 & -0.00253 & -0.203 & -0.00599 & -0.780 & 0.00360 & 0.285 \\
\hline-6 & 0.00164 & 0.277 & -0.00088 & -0.061 & 0.00371 & 0.465 & 0.00732 & 0.497 \\
\hline-5 & $-0.01281 * *$ & -2.116 & -0.01370 & -0.747 & -0.00982 & -1.528 & -0.00250 & -0.131 \\
\hline-4 & -0.00605 & -1.039 & -0.01976 & -0.989 & -0.00514 & -0.939 & -0.00764 & -0.371 \\
\hline-3 & -0.00233 & -0.472 & -0.02210 & -1.165 & -0.00357 & -0.734 & -0.01122 & -0.549 \\
\hline-2 & -0.01108 & -1.519 & $-0.03318 *$ & -1.876 & -0.00904 & -1.283 & -0.02026 & -1.057 \\
\hline-1 & -0.00096 & -0.166 & $-0.03415^{*}$ & -1.822 & 0.00161 & 0.283 & -0.01865 & -0.926 \\
\hline 0 & -0.00560 & -0.993 & $-0.03975^{*}$ & -1.894 & -0.00626 & -1.136 & -0.02492 & -1.135 \\
\hline 1 & 0.00689 & 1.223 & -0.03285 & -1.620 & 0.00792 & 1.466 & -0.01699 & -0.804 \\
\hline 2 & -0.00228 & -0.418 & $-0.03514^{*}$ & -1.797 & -0.00108 & 0.204 & -0.01807 & -0.905 \\
\hline 3 & $0.01165^{* *}$ & 2.074 & -0.02348 & -1.204 & $0.01327 * *$ & 2.334 & -0.00480 & -0.238 \\
\hline 4 & 0.00792 & 1.307 & -0.01555 & -0.756 & $0.01074 *$ & 1.819 & 0.00596 & 0.290 \\
\hline 5 & -0.00677 & -1.107 & -0.02233 & -1.039 & -0.00443 & -0.753 & 0.00153 & 0.069 \\
\hline 6 & 0.00442 & 0.805 & -0.01791 & -0.740 & 0.00594 & 1.092 & 0.00747 & 0.310 \\
\hline 7 & -0.00615 & -1.094 & -0.02407 & -1.075 & -0.00429 & -0.811 & 0.00317 & 0.139 \\
\hline 8 & 0.00319 & 0.741 & -0.02088 & -0.899 & 0.00550 & 1.284 & 0.00868 & 0.378 \\
\hline 9 & 0.00433 & 0.660 & -0.01654 & -0.680 & 0.00566 & 0.865 & 0.01435 & 0.591 \\
\hline 10 & -0.00046 & -0.070 & -0.01700 & -0.655 & 0.00072 & 0.109 & 0.01507 & 0.577 \\
\hline
\end{tabular}

$* * *, * *$ and $*$ denote respectively significance at $1 \%, 5 \%$ and $10 \%$ levels.

To test the crisis impact on investors' reaction to expected versus surprise ratings, we apply ARs and CARs mean difference tests between the two contexts (crisis and stability).

Table 7a displays ARs and CARs mean differences of expected upgrades during crisis and non-crisis periods. Mean CAR differences are only significant the day $+2(-22,9 \%)$ and day +3 $(-23,1 \%)$. This result puts evidence of the intensification adverse reaction of market to expected good news during the crisis period. 
Table 7a. Mean difference tests between ARs and CARs following expected good news during crisis versus stable period

\begin{tabular}{|c|c|c|c|c|c|c|c|c|}
\hline & Stock Index & Adjuste & d Model & & Market Return & Adjuste & d Model & \\
\hline Day $(\mathrm{t})$ & AR & $\mathrm{t}$-stat & CAR & $\mathrm{t}$-stat & AR & $\mathrm{t}$-stat & CAR & $\mathrm{t}$-stat \\
\hline-10 & 0.00391 & 0.148 & 0.00391 & 0.148 & 0.00308 & 0.114 & 0.00308 & 0.114 \\
\hline-9 & -0.02118 & -0.659 & -0.01726 & -0.296 & -0.01948 & -0.744 & -0.01640 & -0.309 \\
\hline-8 & -0.00342 & -0.224 & -0.02069 & -0.409 & -0.00345 & -0.245 & -0.01985 & -0.419 \\
\hline-7 & -0.04262 & -2.494 & -0.06331 & -1.427 & -0.03334 & -1.411 & -0.05319 & -0.978 \\
\hline-6 & -0.06596 & -2.093 & -0.12927 & -1.762 & -0.06620 & -2.705 & -0.11940 & -1.634 \\
\hline-5 & 0.04091 & 1.029 & -0.08836 & -1.156 & 0.03908 & 0.979 & -0.08031 & -1.045 \\
\hline-4 & -0.04463 & -0.982 & $-0.13299 * *$ & -3.960 & -0.03964 & -0.926 & $-0.11996 * *$ & -3.112 \\
\hline-3 & 0.01316 & 0.551 & $-0.11983 * * *$ & -7.297 & 0.01303 & 0.605 & $-0.10692 * * *$ & -4.184 \\
\hline-2 & -0.05577 & -1.389 & $-0.17560 * *$ & -3.729 & -0.04116 & -1.072 & -0.04422 & -2.521 \\
\hline-1 & $-0.02388 * *$ & -2.371 & $-0.19949 *$ & -3.573 & -0.01885 & -1.018 & -0.16695 & -2.188 \\
\hline 0 & -0.02751 & -1.056 & -0.22700 & -2.798 & -0.01782 & -0.838 & -0.18478 & -1.912 \\
\hline 1 & 0.00666 & 0.136 & $-0.22033^{*}$ & -2.669 & 0.01155 & 0.284 & -0.17322 & -1.785 \\
\hline 2 & -0.00953 & -0.250 & $-0.22987 * *$ & -5.077 & -0.01517 & -0.517 & -0.18839 & -2.543 \\
\hline 3 & -0.00144 & -0.052 & $-0.23131 * *$ & -4.505 & -0.01284 & -1.128 & -0.20123 & -2.660 \\
\hline 4 & 0.03717 & 1.210 & -0.19413 & -2.739 & 0.03958 & 1.344 & -0.16165 & -1.886 \\
\hline 5 & -0.03814 & -1.342 & $-0.23228^{*}$ & -3.421 & -0.02613 & -1.785 & -0.18779 & -2.441 \\
\hline 6 & -0.01848 & -1.586 & $-0.25076^{*}$ & -3.200 & -0.02716 & 1.711 & -0.21495 & -2.493 \\
\hline 7 & -0.01623 & -0.695 & $-0.26699 *$ & -3.598 & -0.01404 & -0.589 & -0.22900 & -2.442 \\
\hline 8 & -0.03728 & -0.829 & $-0.30428^{*}$ & -3.237 & -0.03159 & -1.014 & -0.26059 & -2.414 \\
\hline 9 & $0.04197 * *$ & 4.131 & $-0.26231^{*}$ & -3.075 & $0.03954 * * *$ & 5.813 & -0.22105 & -1.989 \\
\hline 10 & $-0.05838 * *$ & -5.296 & $-0.32069 *$ & -3.654 & -0.04877 & -2.410 & -0.26982 & -2.176 \\
\hline
\end{tabular}

$* * *, * *$ and $*$ denote respectively significance at $1 \%, 5 \%$ and $10 \%$ levels.

Results of surprise upgrades are however different. Table 7b relates ARs and CARs mean differences of surprise good news between the two periods. Results show no differences for both of crisis and normal periods, i.e. market reaction to surprise upgrades does not appear to change during the crisis.

Globally, results for good news show no significant reaction to expected upgrades during crisis period, unlike surprise ones which have a very short and positive impact on stock prices. 
Table 7b. Mean difference tests between ARs and CARs following surprise good news during crisis versus stable period

\begin{tabular}{|c|c|c|c|c|c|c|c|c|}
\hline \multirow[b]{2}{*}{ Day $(t)$} & \multicolumn{4}{|c|}{ Stock Index Adjusted Model } & \multicolumn{4}{|c|}{ Market Return Adjusted Model } \\
\hline & AR & $\mathrm{t}$-stat & CAR & $\mathrm{t}$-stat & AR & $\mathrm{t}$-stat & CAR & $\mathrm{t}$-stat \\
\hline-10 & -0.02394 & -0.743 & -0.02394 & -0.743 & -0.02268 & -0.735 & -0.02268 & -0.743 \\
\hline-9 & -0.07813 & -0.950 & -0.10207 & -0.904 & -0.06260 & -1.034 & -0.08529 & -0.950 \\
\hline-8 & 0.10816 & 1.132 & 0.00608 & 0.187 & 0.09994 & 1.177 & 0.01465 & 0.517 \\
\hline-7 & -0.00222 & -0.271 & 0.00385 & 0.113 & 0.00459 & 0.471 & 0.01925 & 0.572 \\
\hline-6 & $0.04999 * *$ & 2.830 & $0.05385^{*}$ & -1.978 & $0.05706^{*}$ & 2.009 & $0.07631 * *$ & 2.232 \\
\hline-5 & 0.06836 & 1.027 & 0.12221 & 1.717 & 0.07030 & 1.020 & 0.14662 & 1.535 \\
\hline-4 & -0.00781 & -0.602 & $0.11440 *$ & 1.654 & 0.00904 & 0.562 & 0.15567 & 1.501 \\
\hline-3 & -0.02220 & -0.946 & 0.09219 & 1.482 & -0.01034 & -0.474 & 0.14532 & 1.310 \\
\hline-2 & -0.00739 & -0.762 & 0.08480 & 1.388 & -0.00949 & -0.968 & 0.13583 & 1.208 \\
\hline-1 & -0.02820 & -1.109 & 0.05660 & 1.301 & -0.00438 & -0.476 & 0.13144 & 1.210 \\
\hline 0 & 0.06563 & 1.332 & 0.12223 & 1.439 & 0.06914 & 1.298 & 0.20059 & 1.256 \\
\hline 1 & 0.00484 & 0.487 & 0.12707 & 1.470 & -0.02620 & -0.888 & 0.17438 & 1.310 \\
\hline 2 & -0.00060 & -0.039 & 0.12647 & 1.303 & 0.00125 & 0.084 & 0.17564 & 1.217 \\
\hline 3 & 0.00769 & 0.462 & 0.13416 & 1.280 & 0.03727 & 1.035 & 0.21292 & 1.212 \\
\hline 4 & -0.00133 & -0.103 & 0.13282 & 1.235 & -0.01583 & -1.381 & 0.19708 & 1.153 \\
\hline 5 & 0.00258 & 0.199 & 0.13541 & 1.359 & -0.00612 & -0.444 & 0.19096 & 1.169 \\
\hline 6 & 0.00122 & 0.125 & 0.13664 & 1.416 & -0.0 & -0.606 & 0.17936 & 1.224 \\
\hline 7 & 0.01243 & 0.849 & 0.14908 & 1.514 & 0.01395 & 0.693 & 0.19331 & 1.221 \\
\hline 8 & 0.00688 & 0.978 & 0.1 & 1.579 & 0.01975 & 1.044 & 0.21306 & 1.218 \\
\hline 9 & $-0.02259 *$ & -2.007 & 0.13337 & 1.327 & -0.01654 & -1.164 & 0.19651 & 1.119 \\
\hline 10 & 0.01004 & 0.709 & 0.14341 & 1.330 & 0.01301 & 0.677 & 0.20952 & 1.091 \\
\hline
\end{tabular}

$* * *, * *$ and $*$ denote respectively significance at $1 \%, 5 \%$ and $10 \%$ levels.

\subsubsection{Impact of Expected Versus Surprise Assertions During Crisis Period}

Figures 9 and 10 show that expected assertions have a long negative impact on stock prices during crisis period (Note 8). In fact, ARs appear the day $0(-7.4 \%)$ and day $+1(-5.6 \%)$. CARs extend from the day $-7(-6.8 \%)$ to day $+10(-27.7 \%)$. Reversely, surprise affirmations generate a very short negative reaction around the announcement day, with significant ARs during the day $-2(-2 \%)$, day $+1(-1,8 \%)$ and day $+2(-2.7 \%)$; and significant CARs from going from the day $-2(6,1 \%)$ to day $0(7.4 \%)$. Results affirm once again the second hypothesis. 


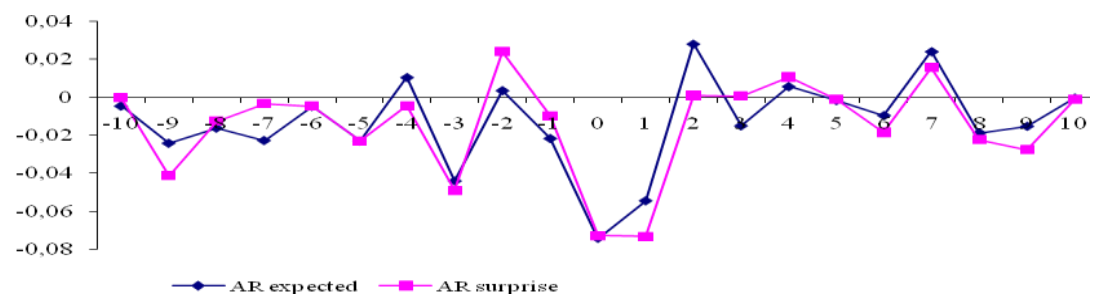

Figure 9. ARs following assertions during crisis period

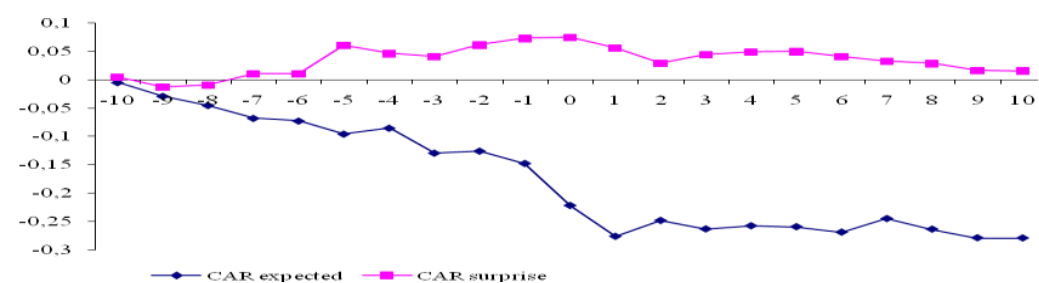

Figure 10. CARs following assertions during crisis period

Reactions are different during stable period (figures 11 and 12). CARs of expected assertions appear from day $-10(-1 \%)$ to day $-4(-2,1 \%)$, and three days after (day $+3(-4,2 \%)$ ). However, surprise affirmations have no impact on stock prices.

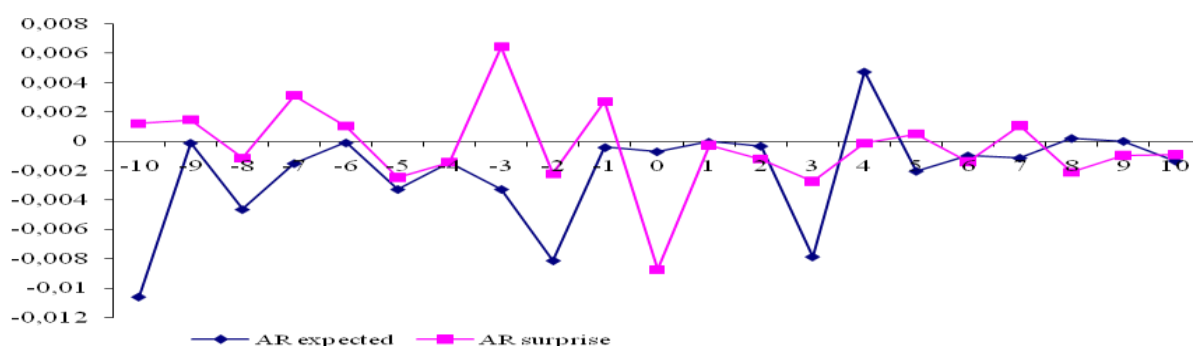

Figure 11. ARs following assertions during stable period

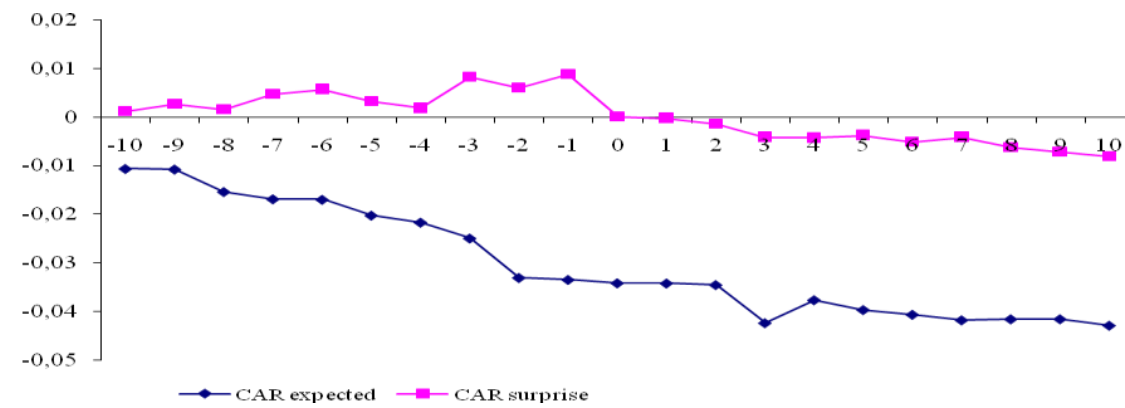

Figure 12. CARs following assertions during stable period 
Consequently, assertions have no impact on stock prices during a normal period. By contrast, expected rating affirmations turn to have negative effects on stock prices during turmoil. In fact, they reveal the maintaining of the priority position of creditors toward stockholders; which explains the investors' adverse reaction.

Table 8a displays the mean difference tests of ARs and CARs following expected and surprise affirmations during crisis period.

Results show that AR mean differences are significant the day $0(-0.7 \%)$ and day $+2(5,5 \%)$. Considering CARs, significant mean differences extend from the day $-3(-17 \%)$ to day +10 $(-29.5 \%)$. This result highlights that market reaction to expected affirmations is higher during crisis period. Investors' lack of confidence in rating agencies is once again highlighted. In fact, investors have confidence in news that have been expected and maturely reflected than those that are only transmitted by rating agencies.

Table 8a. Mean difference tests between ARs and CARs following expected versus surprise assertions during crisis period

\begin{tabular}{|c|c|c|c|c|c|c|c|c|}
\hline & Stock In & $J$ & ted Model & & Market F & $j u$ & Model & \\
\hline $\operatorname{Day}(\mathrm{t})$ & AR & $\mathrm{t}$-stat & CAR & $\mathrm{t}$-stat & $\mathrm{AR}$ & -stat & CAR & $\mathrm{t}$-stat \\
\hline-10 & -0.00 & -0.529 & -0.008 & -0.529 & 374 & .824 & -0.01374 & -0.824 \\
\hline-9 & -0.00712 & 227 & -0.0159 & -0.591 & -0.00308 & .134 & -0.01682 & 0.643 \\
\hline-8 & 6 & 117 & -0.03 & 9 & م 0 & 3 & -0.0 & 0.830 \\
\hline-7 & $-0.0422 *$ & -2.077 & $-0.0780 * *$ & -2.435 & -0.01234 & -0.906 & -0.03760 & -1.282 \\
\hline-6 & -0.00473 & -0.265 & $-0.082 * *$ & -2.2 & -0.01289 & -0.731 & -0.05050 & -1.503 \\
\hline-5 & -0.07430 & -1.374 & $-0.157 * * *$ & -2.853 & -0.04649 & -0.897 & $-0.09700^{*}$ & -1.908 \\
\hline-4 & 0.02550 & 1.309 & $-0.1315^{* *}$ & -2.610 & $0.03548 *$ & 1.940 & -0.06151 & -1.276 \\
\hline-3 & $-0.03915^{*}$ & -1.916 & $-0.17 * * *$ & -3.331 & -0.03122 & -1.473 & $-0.09274 *$ & -1.882 \\
\hline-2 & -0.01678 & -0.583 & $-0.187 * * *$ & -3.127 & -0.02435 & -0.877 & $-0.11709 * *$ & -1.956 \\
\hline-1 & $-0.03358 *$ & -1.791 & $-0.221 * * *$ & -3.232 & -0.03749 & -1.674 & $-0.15459 * *$ & -2.332 \\
\hline 0 & $-0.0758 * *$ & -2.465 & $-0.296 * * *$ & -4.284 & $-0.07738^{* *}$ & -2.583 & $-0.2319 * * *$ & -3.377 \\
\hline 1 & -0.03599 & -1.572 & $-0.332 * * *$ & -4.652 & -0.02439 & -1.100 & $-0.2563 * * *$ & -3.681 \\
\hline 2 & $0.05530 *$ & 2.029 & $-0.277 * * *$ & -4.055 & $0.04453 *$ & 1.791 & $-0.2118 * * *$ & -3.014 \\
\hline 3 & -0.03061 & -0.915 & $-0.308 * * *$ & -4.116 & -0.02154 & -0.698 & $-0.23338 * * *$ & -2.857 \\
\hline 4 & 0.00088 & 0.041 & $-0.30 * * *$ & -4.152 & 0.00517 & 0.290 & $-0.22820 * * *$ & -2.846 \\
\hline 5 & -0.00212 & -0.098 & $-0.309 * * *$ & -4.543 & 0.00712 & 0.350 & $-0.2210 * * *$ & -3.043 \\
\hline 6 & -0.00067 & -0.039 & $-0.310 * * *$ & -4.567 & 0.00741 & 0.570 & $-0.2136 * * *$ & -2.958 \\
\hline 7 & 0.03252 & 1.272 & $-0.277 * * *$ & -3.367 & 0.03405 & 1.402 & $-0.17962 * *$ & -2.086 \\
\hline 8 & -0.01512 & -1.093 & $-0.292 * * *$ & -3.619 & -0.01144 & -0.815 & $-0.19106^{* *}$ & -2.306 \\
\hline
\end{tabular}




\begin{tabular}{lllllllll}
9 & -0.00292 & -0.135 & $-0.295^{* * *}$ & -3.337 & -0.00081 & -0.045 & $-0.19187 * *$ & -2.129 \\
10 & 0.00047 & 0.017 & $-0.295^{* * *}$ & -3.266 & -0.01057 & -0.390 & $-0.20263 * *$ & -2.160 \\
\hline
\end{tabular}

$* * *, * *$ and $*$ denote respectively significance at $1 \%, 5 \%$ and $10 \%$ levels.

Table 8b. Mean Difference tests between ARs and CARs following expected versus surprise assertions during stable period

\begin{tabular}{|c|c|c|c|c|c|c|c|c|}
\hline \multirow[b]{2}{*}{ Day $(t)$} & \multicolumn{4}{|c|}{ Stock Index Adjusted Model } & \multicolumn{4}{|c|}{ Market Return Adjusted Model } \\
\hline & AR & $\mathrm{t}$-stat & CAR & $\mathrm{t}$-stat & AR & $\mathrm{t}$-stat & CAR & $\mathrm{t}$-stat \\
\hline-10 & -0.011 & 830 & -0.011 & -1.830 & $-0.01080^{*}$ & -1.785 & $-0.01080^{*}$ & -1.785 \\
\hline-9 & -0.00157 & -0.413 & -0.01341 & -1.400 & -0.00110 & -0.308 & -0.01190 & -1.297 \\
\hline-8 & -0.00350 & -1.076 & -0.01691 & -1.653 & -0.00151 & -0.446 & -0.01342 & -1.329 \\
\hline-7 & -0.00463 & -1.592 & $-0.02155^{* *}$ & -2.447 & -0.00253 & -0.893 & $-0.01596^{*}$ & -1.834 \\
\hline-6 & -0.00114 & -0.364 & $-0.02269 * *$ & -2.376 & -0.00195 & -0.621 & $-0.01791 *$ & -1.923 \\
\hline-5 & -0.00081 & -0.179 & $-0.0235 * *$ & -2.124 & -0.00052 & -0.118 & $-0.01844^{*}$ & -1.722 \\
\hline-4 & -0.00002 & -0.006 & $-0.02353^{* *}$ & -2.049 & 0.00093 & 0.208 & -0.01751 & -1.521 \\
\hline-3 & $-0.00971 * *$ & -2.046 & $-0.03325^{* *}$ & -2.431 & $-0.00923 *$ & -1.872 & $-0.02674^{*}$ & -1.953 \\
\hline-2 & -0.00592 & -0.590 & $-0.03917 *$ & -1.940 & -0.00612 & -0.610 & -0.03286 & -1.571 \\
\hline-1 & -0.00313 & -0.635 & $-0.04231 * *$ & -2.091 & -0.00276 & -0.539 & $-0.03562 *$ & -1.698 \\
\hline 0 & 0.00767 & 1.353 & -0.03463 & -1.674 & 0.00857 & 1.537 & -0.02705 & -1.259 \\
\hline 1 & 0.00058 & 0.130 & -0.03404 & -1.647 & 0.00301 & 0.675 & -0.02403 & -1.118 \\
\hline 2 & 0.00088 & 0.256 & -0.03316 & -1.652 & 0.00191 & 0.568 & -0.02212 & -1.057 \\
\hline 3 & -0.00515 & -1.373 & $-0.03831 *$ & -1.919 & -0.00327 & -0.855 & -0.02539 & -1.198 \\
\hline 4 & $0.00487 *$ & 1.754 & -0.03344 & -1.687 & $0.00674 * *$ & 2.375 & -0.01864 & -0.877 \\
\hline 5 & 0.00017 & 0.063 & $-0.03597 *$ & -1.740 & -0.00252 & -0.787 & -0.01896 & -0.856 \\
\hline 6 & 0.00041 & 0.125 & $-0.03555^{*}$ & -1.724 & 0.00285 & 0.912 & -0.01610 & -0.729 \\
\hline 7 & -0.00220 & -0.429 & $-0.03776^{*}$ & -1.747 & -0.00187 & -0.436 & -0.01798 & -0.775 \\
\hline 8 & 0.00231 & 0.674 & -0.03544 & -1.553 & 0.00203 & 0.596 & -0.01594 & -0.654 \\
\hline 9 & 0.00097 & 0.293 & -0.03447 & -1.453 & 0.00268 & 0.853 & -0.01326 & -0.533 \\
\hline 10 & -0.00041 & -0.129 & -0.03489 & -1.414 & 0.00019 & 0.066 & -0.01306 & -0.504 \\
\hline
\end{tabular}

$* * *, * *$ and $*$ denote respectively significance at $1 \%, 5 \%$ and $10 \%$ levels.

However, differences between ARs and CARs following expected versus surprise assertions are not significant during stable period (table $8 \mathrm{~b}$ ). This result is in harmony with the findings of Purda (2007) and is explained by the neutral character of assertions. They don't vehicle new information to investors. 
Considering crisis impact on expected affirmations, table 9a displays ARs and CARs mean difference tests. Results show that crisis intensifies market reaction to expected assertions. ARs differences are significant the day $0(-7,3 \%)$ and day $+1(-5,4 \%)$; significant CARs differences extend from the day $0(-18,7 \%)$ to day $+10(-23,6 \%)$.

Table 9a. Mean difference tests between ARs and CARs following expected assertions during crisis versus stable period

\begin{tabular}{|c|c|c|c|c|c|c|c|c|}
\hline \multirow[b]{2}{*}{$\operatorname{Day}(\mathrm{t})$} & \multicolumn{4}{|c|}{ Stock Index Adjusted Model } & \multicolumn{4}{|c|}{ Market Return Adjusted Model } \\
\hline & AR & $\mathrm{t}$-stat & CAR & $\mathrm{t}$-stat & $\mathrm{AR}$ & $\mathrm{t}$-stat & CAR & $\mathrm{t}$-stat \\
\hline-10 & 0.00600 & 0.362 & 0.00600 & 0.362 & -0.00219 & -0.135 & -0.00219 & -0.135 \\
\hline-9 & -0.02410 & -1.105 & -0.01809 & -0.676 & -0.01500 & -0.687 & -0.01719 & -0.678 \\
\hline-8 & -0.01160 & -0.770 & -0.0297 & -0.992 & 0.00009 & 0.005 & -0.01709 & -0.593 \\
\hline-7 & -0.02129 & -1.119 & $-0.05099 *$ & -1.741 & 0.00132 & 0.117 & -0.01577 & -0.590 \\
\hline-6 & -0.00467 & -0.293 & -0.05566 & -1.637 & -0.01261 & -0.790 & -0.02838 & -0.914 \\
\hline-5 & -0.01981 & 0.615 & -0.07548 & -2.041 & 0.00665 & 0.236 & -0.02173 & -0.742 \\
\hline-4 & 0.01189 & 0.782 & -0.06359 & -1.677 & 0.02038 & 1.398 & -0.00135 & -0.040 \\
\hline-3 & $-0.04087 * *$ & -2.188 & $-0.10446 * *$ & -2.446 & -0.03036 & -1.532 & -0.03172 & -0.810 \\
\hline-2 & 0.01166 & 0.394 & $-0.09208^{*}$ & -1.654 & 0.00506 & 0.176 & -0.02665 & -0.479 \\
\hline-1 & -0.02134 & -1.388 & $-0.11414^{*}$ & -1.799 & -0.02723 & -1.416 & -0.05388 & -0.847 \\
\hline 0 & $-0.07368 * *$ & -2.500 & $-0.187 * * *$ & -2.928 & $-0.08059 * *$ & -2.800 & $-0.1344 * *$ & -2.151 \\
\hline 1 & $-0.05459 * *$ & -2.604 & $-0.2424 * * *$ & -3.663 & $-0.04402 * *$ & -2.149 & $-0.1785^{* *}$ & -2.812 \\
\hline 2 & 0.02842 & 1.143 & $-0.2140 * * *$ & -3.324 & 0.01377 & 0.610 & $-0.16472 * *$ & -2.530 \\
\hline 3 & -0.00716 & -0.218 & $-0.2216^{* * *}$ & -3.143 & 0.00032 & 0.010 & $-0.164 * *$ & -2.145 \\
\hline 4 & 0.00089 & 0.046 & $-0.220 * * *$ & -3.119 & 0.00477 & 0.310 & $-0.15962 *$ & -2.102 \\
\hline 5 & 0.00033 & 0.017 & $-0.22 * * *$ & -3.348 & 0.00486 & 0.276 & $-0.1547 * *$ & -2.213 \\
\hline 6 & -0.00863 & -0.552 & $-0.228 * * *$ & -3.491 & 0.00014 & 0.013 & $-0.1546^{* *}$ & -2.232 \\
\hline 7 & 0.02520 & 1.178 & $-0.2033 * *$ & -2.571 & 0.02459 & 1.228 & -0.13002 & -1.580 \\
\hline 8 & -0.01898 & -1.567 & $-0.2223 * *$ & -2.889 & -0.01628 & -1.310 & $-0.14630^{*}$ & -1.858 \\
\hline 9 & -0.01528 & -0.768 & $-0.2376^{* *}$ & -2.786 & -0.01778 & -1.114 & $-0.16408^{*}$ & -1.914 \\
\hline 10 & 0.00097 & 0.037 & $-0.23665^{* *}$ & -2.717 & -0.00897 & -0.337 & $-0.17306^{*}$ & -1.946 \\
\hline
\end{tabular}

$* * *, * *$ and $*$ denote respectively significance at $1 \%, 5 \%$ and $10 \%$ levels.

Globally, we find similar results as for bad rating news. Crisis slightly impacts market reaction to surprise assertions. These results show once again the weak impact of assertions on stock markets, and the cautious attitude of investors toward rating agencies. 
Table 9b. Mean difference tests between ARs and CARs following surprise assertions during crisis versus stable period

\begin{tabular}{|c|c|c|c|c|c|c|c|c|}
\hline \multicolumn{5}{|c|}{ Stock Index Adjusted Model } & \multicolumn{4}{|c|}{ Market Return Adjusted Model } \\
\hline Day $(\mathrm{t})$ & AR & $\mathrm{t}$-stat & CAR & $\mathrm{t}$-stat & AR & $\mathrm{t}$-stat & CAR & $\mathrm{t}$-stat \\
\hline-10 & 0.00299 & 0.390 & 0.00299 & 0.390 & 0.00074 & 0.090 & 0.00074 & 0.090 \\
\hline-9 & $-0.0185^{* *}$ & -2.339 & -0.01551 & -1.537 & -0.01301 & -1.644 & -0.01227 & -1.100 \\
\hline-8 & 0.00475 & 0.482 & -0.01081 & $\operatorname{tr}$ & 0.00702 & 0.739 & -0.00525 & -0.327 \\
\hline-7 & $0.01628 * *$ & 2.083 & 0.00547 & 0.348 & 0.01112 & 1.348 & 0.00587 & 0.393 \\
\hline-6 & -0.00107 & -0.126 & 0.00439 & 0.247 & -0.00166 & -0.205 & 0.00420 & 0.265 \\
\hline-5 & 0.05366 & 1.227 & 0.05806 & 1.373 & 0.05262 & 1.202 & 0.05682 & 1.324 \\
\hline-4 & -0.01364 & -1.063 & 0.04441 & 1.264 & -0.01417 & -1.196 & 0.04265 & 1.160 \\
\hline-3 & -0.01143 & -1.212 & 0.03298 & 1.049 & -0.00837 & -0.946 & 0.03428 & 1.041 \\
\hline-2 & $0.02252 * *$ & 2.473 & $0.05550^{*}$ & 1.895 & $0.0233 * * *$ & 2.696 & 0.05757 & 1.897 \\
\hline-1 & 0.00911 & 0.779 & $0.0646^{*}$ & 1.981 & 0.00749 & 0.602 & $0.06507 *$ & 1.885 \\
\hline 0 & 0.00986 & 0.954 & $0.0745 * *$ & 2.226 & 0.00536 & 0.546 & $0.07044 *$ & 1.973 \\
\hline 1 & $-0.01802^{*}$ & -1.766 & 0.05646 & 1.651 & $-0.01660 *$ & -1.746 & 0.05383 & 1.501 \\
\hline 2 & $-0.02598 * *$ & -2.227 & 0.03047 & 0.991 & $-0.028 * * *$ & -2.651 & 0.02499 & 0.740 \\
\hline 3 & $0.01829 * *$ & 2.281 & 0.04876 & 1.500 & $0.01859 * *$ & 2.133 & 0.04358 & 1.232 \\
\hline 4 & 0.00488 & 0.524 & $0.05365^{*}$ & 1.804 & 0.00635 & 0.676 & 0.04993 & 1.494 \\
\hline 5 & -0.00006 & -0.005 & $0.05359 *$ & 1.953 & -0.00257 & -0.241 & 0.04736 & 1.595 \\
\hline 6 & -0.00754 & -0.972 & 0.04604 & 1.677 & -0.00440 & -0.602 & 0.04295 & 1.425 \\
\hline 7 & -0.00951 & -0.651 & 0.03652 & 1.149 & -0.01133 & -0.792 & 0.03161 & 0.919 \\
\hline 8 & -0.00154 & -0.203 & 0.03498 & 1.036 & -0.00280 & -0.382 & 0.02881 & 0.812 \\
\hline 9 & -0.01139 & -1.245 & 0.02359 & 0.697 & -0.01429 & -1.617 & 0.01452 & 0.389 \\
\hline 10 & 0.00007 & 0.008 & 0.02367 & 0.685 & 0.00197 & 0.243 & 0.01650 & 0.417 \\
\hline
\end{tabular}

$* * *, * *$ and $*$ denote respectively significance at $1 \%, 5 \%$ and $10 \%$ levels.

Globally, expected assertions during crisis period have stronger impact on stock prices than surprise ones. This result is in line with bad news findings. Investors' behavior is guided by risk aversion and carefulness towards rating agencies. Also, crisis amplifies impact of expected neutral news and has no impact on surprise ones.

\section{Conclusion}

In this paper, we study the differential impact of surprise versus expected rating announcements on stock prices during 2008 financial crisis. Expected ratings are preceded by 
significant abnormal returns. We collect 216 rating announcements during the American financial crisis period, going from 16th September to 31st December 2008. We follow these firms' ratings during the stable period which extends from January 2003 to December 2006. We calculate for each announcement the cumulative abnormal return (CAR) over 120 listing days preceding the rating event and test for its significance. Announcements with significant CARs are considered as expected ratings, the others are surprises. The correlation test between crisis and calculated CARs shows that there is a significant relation between crisis and expected ratings for both of the bad and the good news. This is not valid for the assertions. Expected downgrades and upgrades are more accentuated during crisis period unlike expected assertions. Then, for each announcement, we apply event study which consists in calculating ARs and CARs over 20 days symmetrically around rating announcement. We test for their significance and apply mean difference tests to calculate firstly difference in reaction between expected and unexpected ratings for both of crisis and stable periods, and secondly to measure the impact of crisis on reaction to expected and surprise ratings. Results are similar for bad and neutral rating news, on contrary to good ones. In fact, expected bad and neutral rating announcements generate stronger reaction during crisis period. Also, crisis enhances reaction to expected announcements and does not for surprise ones. As regards good news, expected announcements have no impact on stock prices during crisis period; in contrast surprise ratings have weak and short effect on stock prices. Besides, crisis slightly intensifies impact of expected good news, and does not for surprise good announcements.

Globally, this study shows evidence of the cautious investors' behavior towards rating announcements during crisis period. They trustful expected bad and neutral ratings and are skeptical towards informations solely diffused by rating agencies. These have to revise their methodologies and procedures in order to recover place on financial markets. However, White (2010) showed that regulation enhances barriers to entry and inhibits innovation. Hence, prudential regulation of rating opinions should be reformed to maintain agencies' independence and to improve financial market efficiency.

\section{References}

Bahena, A. J. (2010). What Role Did Credit Rating Agencies Play in the Credit Crisis? Global Money, the Good Life, and You. Understanding the Local Impact of International Financial Institutions.

Brown, S. J., \& Warner, J. B. (1985). Using daily stock returns: The case of event studies. Journal of Financial Economics, 14, 3-31.

Cornaggia, J., Cornaggia, K. J., \& Hund, J. E. (2017). Credit rating across asset classes: a long term perspective. Review of Finance, 21(2), 465-509.

DeHaan, E. (2017). The Financial Crisis and Corporate Credit Ratings. Accounting Review, 92(84), 161-189.

Di Cesare, A. (2006). Do market based indicators anticipate rating agencies? Evidence for international banks. Working Paper, Temi di discussione del servizio study, Banca d'Italia, N 
593.

Ederington, L. H., \& Goh, J. C. (1993). Is a bond rating downgrade bad news, good news or no news for stockholders?. The Journal of Finance, 48(5), 2001-2008.

Gallo, G. M., \& Velucchi, M. (2009). Market Interdependence and Financial Volatility Transmission in East Asia. International Journal of Financial and Economics, 14, 24-44.

Glascock, J. L., Wallace, N. D., \& Glenn, V. H. (1987). Announcement effects of Moody's bond ratings changes on equity returns. Quarterly Journal of Business and Economics, 26, 67-78.

Grier, P., \& Katz, S. (1976). The differential effects of bond rating changes among industrial and public utility bonds by maturity. Journal of Business, 2(49), 226-239.

Griffin, P., \& Sanvicente, A. (1982). Common stock returns and rating changes: A methodological comparison. The Journal of Finance, 37, 103-119.

Hand, J. R. M., Holthausen, R. W., \& Leftwich, R. W. (1992).The effect of bond rating agency announcements on bond and stock prices. The Journal of Finance, 47(2), 733-752.

Hettenhouse, G. W., \& Sartoris, W. L. (1976). An Analysis of the information value of bond rating changes. Quaterly Review of Economics and Business, 16, 65-78.

Hite, G., \& Warga, A. (1997). The effect of bond rating changes on bond price performance. Financial Analysts Journal, 53, 35-51.

Hull, J., Predescu, M., \& White, A. (2004). The relationship between credit default swap spreads, bond yields and credit rating announcements. Journal of Banking and Finance, 28(11), 2789-2811.

Katz, S. (1973). The price and adjustment process of bonds to rating reclassifications:A test of bond market efficiency. The Journal of Finance, 2(29), 551-559.

Michayluk, D., \& Neuhauser, K. L. (2006). Investor overreaction during market declines: Evidence from the 1997 Asian Financial Crisis. The Journal of Financial Research, 30(2), 217-234.

Micu, M., Remonola, E. M., \& Wooldridge, P. D. (2004). The price impact of rating announcements: evidence from the credit default swap market. Bis Quaterly Review, 5, $55-65$.

Norden, L., \& Weber, M. (2004). Informational efficiency of credit default swap and stock markets: The impact of credit rating announcements. Journal of Banking and Finance, 28, 2813-2843.

Pinches, G. E., \& Singleton, J. C. (1978). The adjustment of stock prices to bond rating changes. The Journal of Finance, 33, 29-44.

Purda, L. D. (2007). Stock Market reaction to anticipated versus surprise rating changes. Journal of Financial Research, 30(2), 301 -320. 


\section{Ml Macrothink}

International Journal of Accounting and Financial Reporting

ISSN 2162-3082 2018, Vol. 8, No. 3

Steiner, M., \& Heinke, V. (2001). Event study concerning international bond price effects of credit rating actions. International Journal of Finance and Economics, 6, 139-157.

Weinstein, M. (1977). The effect of a rating change announcement on bond price. Journal of Financial Economics, 5, 329-350.

White, L. J. (2010). Credit rating agencies and the financial crisis: less regulations of CRAs is a better response. Working Papers 10-03, New York University, Leonard N. Stern School of Business, Department of Economics.

\section{Notes}

Note 1. Results were confirmed by Logit model.

Note 2. Announcements are collected from the rating agencies web sites and the Compustat North America database.

Note 3. Revisions for change are assimilated to changes with zero amplitude.

Note 4. The choice of 120 days for calculating the significant CAR before the rating announcement is currently adopted in literature relatively to anticipated notation.

Note 5 . The $\mathrm{Z}$ test gives the probability that an observation is significantly different from the sample mean to which it belongs.

Note 6. Results of ARs and CARs following expected and surprise bad rating news during crisis and non-crisis period are available in appendix 1.

Note 7. Results of ARs and CARs following expected and surprise good rating news during crisis and non-crisis period are available in appendix 2.

Note 8. Results of ARs and CARs following expected and surprise neutral rating news during crisis and non-crisis period are available in appendix 3. 


\section{Appendix 1}

ARs and CARs following surprise bad rating news during crisis period

\begin{tabular}{|c|c|c|c|c|c|c|c|c|}
\hline \multirow[b]{2}{*}{$\operatorname{day}(t)$} & \multicolumn{3}{|c|}{ Stock adjusted returns } & \multirow[b]{2}{*}{ t statistic } & \multicolumn{3}{|c|}{$\begin{array}{c}\text { OLS Market model } \\
\text { returns }\end{array}$} & \multirow[b]{2}{*}{ t statistic } \\
\hline & AR & t statistic & CAR & & AR & t statistic & CAR & \\
\hline-10 & -0.00169 & -0.276 & -0.00169 & -0.276 & 0.00359 & 0.472 & 0.00359 & 0.472 \\
\hline-9 & -0.00278 & -0.485 & -0.00448 & -0.505 & 0.00388 & 0.511 & 0.00747 & 0.568 \\
\hline-8 & 0.00175 & -0.244 & -0.00272 & -0.257 & 0.00593 & 0.913 & 0.01341 & 0.913 \\
\hline-7 & 0.00228 & 0.307 & -0.00044 & -0.037 & 0.01142 & 1.121 & 0.02483 & 1.158 \\
\hline-6 & -0.00737 & -1.050 & -0.00781 & -0.564 & -0.00327 & -0.474 & 0.02155 & 0.852 \\
\hline-5 & -0.01002 & -1.172 & -0.01784 & -1.071 & -0.02292 & -1.541 & -0.00137 & -0.072 \\
\hline-4 & -0.00012 & -0.158 & -0.01661 & -0.954 & -0.00021 & -0.027 & -0.00158 & -0.076 \\
\hline-3 & -0.00989 & -1.510 & -0.02651 & -1.484 & 0.00400 & 0.439 & 0.00241 & 0.097 \\
\hline-2 & 0.00717 & 0.885 & -0.01933 & -1.079 & 0.00580 & 0.959 & 0.00822 & 0.361 \\
\hline-1 & -0.00078 & -0.119 & -0.02012 & -1.096 & 0.00034 & 0.053 & 0.00857 & 0.369 \\
\hline 0 & $-0.03662^{* * *}$ & -2.931 & $-0.05675^{* *}$ & -2.514 & $-0.03906^{* *}$ & -2.708 & -0.02849 & -1.144 \\
\hline 1 & -0.00622 & -0.490 & $-0.06297^{* * *}$ & -2.682 & -0.00557 & -0.391 & -0.03404 & -1.247 \\
\hline 2 & -0.00405 & -0.448 & $-0.06702^{* *}$ & -2.531 & 0.00553 & 0.513 & -0.02851 & -0.873 \\
\hline 3 & $-0.02536^{* * *}$ & -3.023 & $-0.09239 * * *$ & -3.391 & $-0.02169 * *$ & -2.381 & -0.05020 & -1.509 \\
\hline 4 & -0.00476 & -0.590 & $-0.09716^{* * *}$ & -3.411 & -0.00136 & -0.165 & -0.05157 & -1.414 \\
\hline 5 & $-0.01863^{* *}$ & -2.549 & $-0.11579 * * *$ & -3.668 & -0.00113 & -1.181 & -0.06289 & -1.500 \\
\hline 6 & 0.00305 & 0.508 & $-0.11274^{* * *}$ & -3.694 & -0.00736 & -0.650 & $-0.07025^{*}$ & -1.971 \\
\hline 7 & 0.00680 & 0.764 & $-0.10593^{* * *}$ & -3.327 & 0.01074 & 1.277 & -0.05951 & -1.622 \\
\hline 8 & 0.00794 & 0.562 & $-0.09799 * * *$ & -3.168 & 0.00697 & 0.484 & -0.05254 & -1.473 \\
\hline 9 & -0.00287 & -0.280 & $-0.10086^{* * *}$ & -3.178 & -0.00442 & -0.434 & -0.05696 & -1.571 \\
\hline 10 & 0.00179 & 0.244 & -0.09906 & 0.002 & 0.00684 & 0.910 & -0.05011 & -1.357 \\
\hline
\end{tabular}

\begin{tabular}{|c|c|c|c|c|c|c|c|c|}
\hline \multicolumn{9}{|c|}{ ARs and CARs following expected bad rating news during crisis period } \\
\hline \multicolumn{4}{|c|}{ Stock adjusted returns } & \multicolumn{4}{|c|}{ OLS Market model returns } & \multirow[b]{2}{*}{ t statistic } \\
\hline $\operatorname{day}(t)$ & $\mathrm{AR}$ & t statistic & CAR & t statistic & $\mathrm{AR}$ & t statistic & $\mathrm{CAR}$ & \\
\hline-10 & -0.00377 & -0.183 & -0.00377 & -0.183 & -0.00337 & -0.168 & -0.00337 & -0.168 \\
\hline-9 & $-0.03008^{*}$ & -1.895 & -0.03385 & -1.129 & -0.02481 & -1.580 & -0.02819 & -0.947 \\
\hline-8 & 0.00950 & 0.571 & -0.02435 & -0.726 & 0.01609 & 0.966 & -0.01209 & -0.361 \\
\hline-7 & -0.00767 & -0.432 & -0.03203 & -0.912 & -0.00915 & -0.546 & -0.02125 & -0.608 \\
\hline-6 & 0.02476 & 0.738 & -0.00727 & -0.172 & 0.02823 & 0.864 & 0.00698 & 0.166 \\
\hline-5 & $-0.03311^{* *}$ & -2.461 & -0.04038 & -0.946 & $-0.03098^{* *}$ & -2.255 & -0.02400 & -0.567 \\
\hline-4 & $-0.03463^{*}$ & -1.951 & $-0.07502^{*}$ & -1.708 & -0.02852 & -1.640 & -0.05252 & -1.198 \\
\hline-3 & $-0.03583^{* *}$ & -2.507 & $-0.11085^{* *}$ & -2.439 & $-0.02874^{* *}$ & -2.045 & $-0.08127^{*}$ & -1.819 \\
\hline-2 & $-0.03111^{*}$ & -1.865 & $-0.14197^{* * *}$ & -2.970 & $-0.03154^{*}$ & -1.925 & $-0.11281^{* *}$ & -2.385 \\
\hline-1 & $-0.08107^{* * *}$ & -3.851 & $-0.22304^{* * *}$ & -4.752 & $-0.07416^{* * *}$ & -3.526 & $-0.18698^{* * *}$ & -3.991 \\
\hline 0 & -0.01500 & -0.538 & $-0.23804^{* * *}$ & -4.686 & -0.00944 & -0.349 & $-0.19642^{* * *}$ & -3.850 \\
\hline 1 & -0.00753 & -0.423 & $-0.24558^{* * *}$ & -4.506 & -0.00755 & -0.445 & $-0.20398^{* * *}$ & -3.712 \\
\hline 2 & -0.00881 & -0.636 & $-0.25440^{* * *}$ & -4.236 & -0.00341 & -0.250 & $-0.20740^{* * *}$ & -3.441 \\
\hline 3 & -0.01732 & -1.117 & $-0.27172^{* * *}$ & -4.361 & -0.01027 & -0.689 & $-0.21767^{* * *}$ & -3.495 \\
\hline 4 & 0.01517 & 1.000 & $-0.25655^{* * *}$ & -4.361 & 0.02340 & 1.583 & $-0.19426^{* * *}$ & -3.291 \\
\hline 5 & 0.01098 & 0.553 & $-0.24556 * * *$ & -3.781 & 0.01532 & 0.811 & $-0.17894^{* * *}$ & -2.752 \\
\hline 6 & -0.00847 & -0.498 & $-0.25404^{* * *}$ & -3.766 & -0.00542 & -0.323 & $-0.18437^{* * *}$ & -2.715 \\
\hline 7 & 0.00207 & 0.094 & $-0.25197^{* * *}$ & -3.386 & 0.00818 & 0.370 & $-0.17618^{* *}$ & -2.339 \\
\hline 8 & -0.00961 & -0.6331 & $-0.26159 * * *$ & -3.488 & -0.00844 & -0.597 & $-0.18463^{* *}$ & -2.434 \\
\hline 9 & 0.03226 & 1.279 & $-0.22932^{* * *}$ & -3.142 & 0.03450 & 1.419 & $-0.15012^{* *}$ & -2.006 \\
\hline 10 & 0.02628 & 1.116 & $-0.20304^{* * *}$ & -2.730 & 0.02839 & 1.218 & -0.12173 & -1.584 \\
\hline
\end{tabular}


International Journal of Accounting and Financial Reporting ISSN 2162-3082 2018, Vol. 8, No. 3

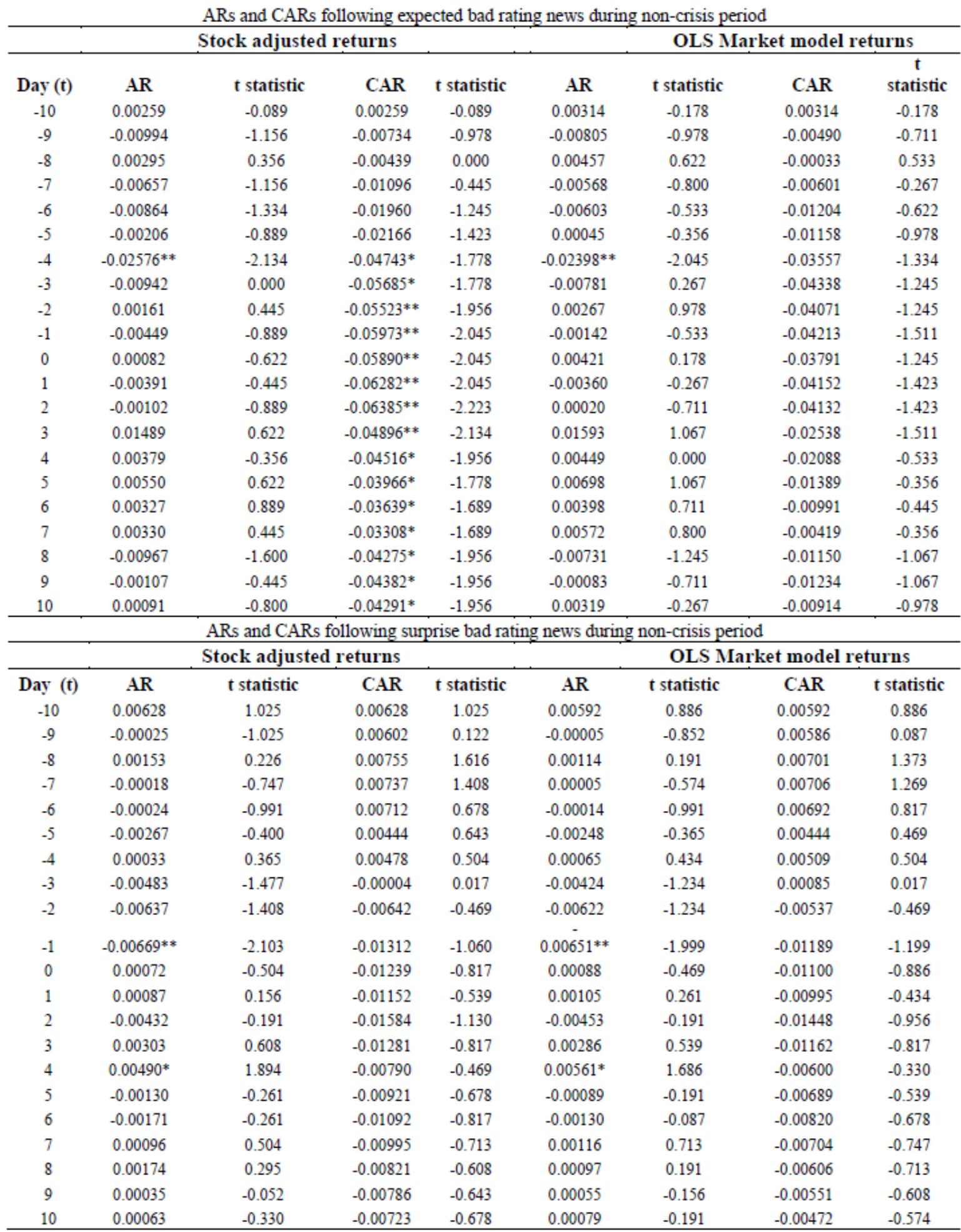

$* * *, * *$ and $*$ denote respectively significance at $1 \%, 5 \%$ and $10 \%$ levels 
Appendix 2

ARs and CARs following expected good rating news during crisis period

\begin{tabular}{|c|c|c|c|c|c|c|c|c|}
\hline \multicolumn{5}{|c|}{ Stock adjusted returns } & \multicolumn{4}{|c|}{ OLS Market model returns } \\
\hline Day (t) & $\mathrm{AR}$ & t statistic & CAR & t statistic & AR & t statistic & CAR & t statistic \\
\hline-10 & 0.00289 & 0.000 & 0.00289 & 0.000 & 0.00369 & 0.000 & 0.00369 & 0.000 \\
\hline-9 & -0.01964 & -0.535 & -0.01674 & 0.000 & -0.01747 & -0.535 & -0.01377 & 0.000 \\
\hline-8 & -0.00934 & 0.000 & -0.02608 & 0.000 & -0.00940 & 0.000 & -0.02318 & 0.000 \\
\hline-7 & -0.04655 & -1.604 & -0.07264 & -1.604 & -0.03771 & -1.069 & -0.06089 & -1.069 \\
\hline-6 & -0.06382 & -1.604 & -0.13646 & -1.604 & -0.06436 & -1.604 & -0.12526 & -1.604 \\
\hline-5 & 0.03561 & 1.069 & -0.10085 & -1.069 & 0.03434 & 0.535 & -0.09091 & -1.069 \\
\hline-4 & -0.04578 & -1.069 & -0.14664 & -1.604 & -0.04071 & -1.069 & -0.13163 & -1.604 \\
\hline-3 & 0.00980 & 0.535 & -0.13683 & -1.604 & 0.00925 & 0.535 & -0.12237 & -1.604 \\
\hline-2 & -0.06238 & -1.604 & -0.19922 & -1.604 & -0.04772 & -1.069 & -0.17010 & -1.604 \\
\hline-1 & -0.02392 & -1.604 & -0.22314 & -1.604 & -0.01904 & -1.069 & -0.18915 & -1.604 \\
\hline 0 & -0.03408 & -1.604 & -0.25722 & -1.604 & -0.02500 & -1.069 & -0.21415 & -1.604 \\
\hline 1 & 0.01552 & 0.535 & -0.24170 & -1.604 & 0.01910 & 0.000 & -0.19505 & -1.604 \\
\hline 2 & -0.00764 & -0.535 & -0.24934 & -1.604 & -0.01381 & -0.535 & -0.20886 & -1.604 \\
\hline 3 & 0.00351 & 0.000 & -0.24582 & -1.604 & -0.00764 & -0.535 & -0.21650 & -1.604 \\
\hline 4 & 0.04426 & 1.604 & -0.20156 & -1.604 & 0.04729 & 1.604 & -0.16920 & -1.604 \\
\hline 5 & -0.04662 & -1.604 & -0.24818 & -1.604 & -0.03388 & -1.604 & -0.20309 & -1.604 \\
\hline 6 & -0.01909 & -1.069 & -0.26727 & -1.604 & -0.02755 & -1.069 & -0.23065 & -1.604 \\
\hline 7 & -0.01422 & -1.069 & -0.28150 & -1.604 & -0.01264 & -0.535 & -0.24329 & -1.604 \\
\hline 8 & -0.03605 & -1.069 & -0.31755 & -1.604 & -0.03022 & -1.069 & -0.27352 & -1.604 \\
\hline 9 & 0.04680 & 1.604 & -0.27074 & -1.604 & 0.04440 & 1.604 & -0.22911 & -1.604 \\
\hline 10 & -0.05775 & -1.604 & -0.32849 & -1.604 & -0.04851 & -1.604 & -0.27763 & -1.604 \\
\hline \multicolumn{9}{|c|}{ ARs and CARs following surprise good rating news during crisis period } \\
\hline \multicolumn{5}{|c|}{ Stock adjusted returns } & \multicolumn{4}{|c|}{ OLS Market model returns } \\
\hline Day (t) & AR & t statistic & CAR & t statistic & AR & t statistic & CAR & t statistic \\
\hline-10 & -0.02751 & -1.007 & -0.02751 & -1.007 & -0.02731 & -1.125 & -0.02731 & -1.125 \\
\hline-9 & -0.08017 & -0.296 & -0.10768 & -1.125 & -0.06677 & -0.770 & -0.09408 & -1.244 \\
\hline-8 & 0.10494 & 0.770 & -0.00274 & -0.059 & 0.09581 & 0.652 & 0.00172 & -0.296 \\
\hline-7 & -0.00019 & 0.059 & -0.00294 & -0.178 & 0.00621 & 0.652 & 0.00794 & -0.415 \\
\hline-6 & $0.05049 * *$ & 2.310 & 0.04755 & 1.481 & $0.05519 * *$ & 2.073 & 0.06313 & 1.244 \\
\hline-5 & 0.07587 & 1.362 & 0.12342 & 1.599 & 0.07539 & 1.244 & 0.13853 & 1.007 \\
\hline-4 & -0.00290 & -0.296 & $0.12052^{*}$ & 1.836 & 0.01311 & 0.889 & 0.15164 & 1.481 \\
\hline-3 & -0.02322 & -0.770 & $0.09729 *$ & 1.836 & -0.01054 & 0.059 & 0.14110 & 1.599 \\
\hline-2 & -0.00291 & -0.059 & 0.09437 & 1.599 & -0.00700 & -0.533 & 0.13409 & 1.362 \\
\hline-1 & -0.02727 & -0.770 & 0.06710 & 1.125 & -0.00618 & -0.770 & 0.12790 & 1.007 \\
\hline 0 & 0.06466 & 1.362 & $0.13177^{* *}$ & 2.192 & 0.06822 & 1.599 & $0.19613^{*}$ & 1.836 \\
\hline 1 & 0.00680 & 0.889 & $0.13857^{* *}$ & 2.192 & -0.02658 & -0.178 & $0.16955^{*}$ & 1.718 \\
\hline 2 & 0.00357 & -0.533 & $0.14214^{*}$ & 1.718 & 0.00369 & 0.059 & 0.17324 & 1.362 \\
\hline 3 & 0.00099 & 0.059 & 0.14314 & 1.362 & 0.02920 & 0.415 & 0.20245 & 1.007 \\
\hline 4 & -0.00218 & 0.296 & 0.14096 & 1.362 & -0.01888 & -1.125 & 0.18356 & 0.415 \\
\hline 5 & 0.00088 & 0.296 & 0.14184 & 1.481 & -0.00944 & -0.415 & 0.17412 & 0.652 \\
\hline 6 & -0.00379 & -0.652 & 0.13805 & 1.481 & -0.01793 & -1.007 & 0.15619 & 0.415 \\
\hline 7 & 0.02060 & 1.007 & $0.15865^{*}$ & 1.718 & 0.01965 & 0.415 & 0.17584 & 0.889 \\
\hline 8 & 0.00493 & 1.362 & $0.16358^{*}$ & 1.718 & 0.01560 & 1.125 & 0.19145 & 1.125 \\
\hline 9 & $-0.02210^{*}$ & -1.836 & 0.14148 & 1.481 & -0.01735 & -1.125 & 0.17409 & 0.415 \\
\hline 10 & 0.01114 & 0.533 & 0.15262 & 1.481 & 0.01254 & 0.296 & 0.18664 & 0.178 \\
\hline
\end{tabular}

${ }^{* * *},{ }^{* *}$ and ${ }^{*}$ denote respectively significance at $1 \%, 5 \%$ and $10 \%$ levels 
ARs and CARs following expected good rating news during non-crisis period

\begin{tabular}{|c|c|c|c|c|c|c|c|c|}
\hline \multicolumn{4}{|c|}{ Stock adjusted returns } & \multicolumn{5}{|c|}{ OLS Market model returns } \\
\hline Day (t) & AR & t statistic & CAR & t statistic & AR & t statistic & CAR & t statistic \\
\hline-10 & -0.00102 & -0.450 & -0.00102 & -0.450 & 0.00061 & -0.071 & 0.00061 & -0.071 \\
\hline-9 & 0.00154 & 0.260 & 0.00052 & 0.213 & 0.00201 & 0.308 & 0.00262 & 0.355 \\
\hline-8 & -0.00592 & -1.254 & -0.00539 & -0.497 & -0.00595 & -1.207 & -0.00332 & -0.260 \\
\hline-7 & -0.00393 & -0.497 & -0.00933 & -0.639 & -0.00437 & -0.544 & -0.00770 & -0.402 \\
\hline-6 & 0.00213 & 0.308 & -0.00719 & -0.497 & 0.00184 & 0.308 & -0.00585 & 0.118 \\
\hline-5 & -0.00530 & -1.160 & -0.01249 & -0.592 & -0.00474 & -0.686 & -0.01059 & -0.213 \\
\hline-4 & -0.00114 & 0.024 & -0.01364 & -0.828 & -0.00107 & -0.024 & -0.01167 & -0.544 \\
\hline-3 & -0.00335 & -0.544 & -0.017005 & -0.734 & -0.00377 & -0.686 & -0.01544 & -0.450 \\
\hline-2 & $-0.00661^{*}$ & -1.681 & -0.02361 & -1.586 & $-0.00655^{* *}$ & -1.775 & -0.02200 & -1.207 \\
\hline-1 & -0.00003 & -0.308 & -0.02365 & -1.491 & -0.00018 & -0.355 & -0.02219 & -1.302 \\
\hline 0 & -0.00657 & -1.018 & $-0.03022^{*}$ & -1.728 & -0.00718 & -1.065 & -0.02937 & -1.444 \\
\hline 1 & $0.00886^{* *}$ & 1.917 & $-0.02136^{*}$ & -1.728 & $0.00754^{*}$ & 1.728 & -0.02183 & -1.349 \\
\hline 2 & 0.00189 & 0.355 & -0.01947 & -1.586 & 0.00136 & 0.355 & -0.02046 & -1.491 \\
\hline 3 & 0.00495 & 0.592 & -0.01451 & -1.302 & 0.00519 & 0.923 & -0.01527 & -1.160 \\
\hline 4 & 0.00708 & 1.112 & -0.00742 & -0.828 & 0.00771 & 1.254 & -0.00755 & -0.828 \\
\hline 5 & $-0.00847 * *$ & -2.012 & -0.01590 & -1.160 & $-0.00775^{* *}$ & -1.965 & -0.01530 & -0.923 \\
\hline 6 & -0.00060 & 0.071 & -0.01650 & -0.923 & -0.00039 & 0.213 & -0.01569 & -1.065 \\
\hline 7 & 0.00200 & 0.781 & -0.01450 & -1.065 & 0.00140 & 0.686 & -0.01429 & -1.065 \\
\hline 8 & 0.00123 & 0.166 & -0.01326 & -0.876 & 0.00136 & -0.024 & -0.01292 & -0.828 \\
\hline 9 & 0.00483 & 0.734 & -0.00845 & -0.544 & 0.00486 & 0.734 & -0.00806 & -0.497 \\
\hline 10 & 0.00062 & -0.213 & -0.00078 & -0.402 & 0.00026 & -0.166 & -0.00780 & -0.450 \\
\hline
\end{tabular}

ARs and CARs following surprise good rating news during non-crisis period

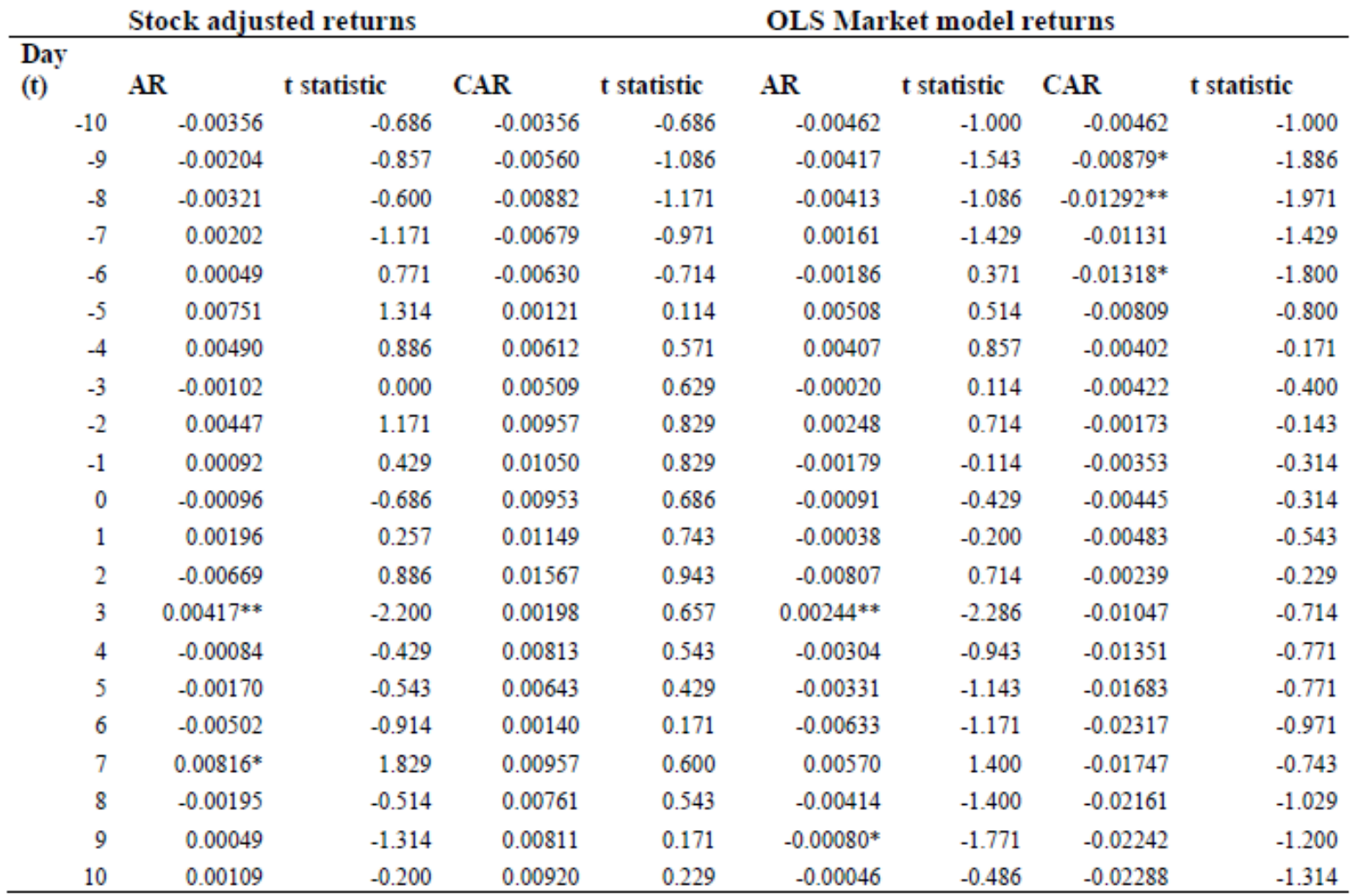

$* * *, * *$ and $*$ denote respectively significance at $1 \%, 5 \%$ and $10 \%$ levels 
Appendix 3

$A R s$ and $C A R s$ following expected neutral rating news during crisis period

\begin{tabular}{|c|c|c|c|c|c|c|c|c|}
\hline \multicolumn{4}{|c|}{ Stock adjusted returns } & \multicolumn{4}{|c|}{ OLS Market model returns } & \multirow[b]{2}{*}{ t statistic } \\
\hline Day (t) & $\mathrm{AR}$ & t statistic & CAR & t statistic & AR & t statistic & CAR & \\
\hline-10 & -0.00461 & -1.241 & -0.00461 & -1.241 & $-0.01263^{*}$ & -1.810 & $-0.01263^{*}$ & -1.810 \\
\hline-8 & -0.01624 & -1.034 & -0.04507 & -1.344 & -0.00405 & -0.259 & -0.03200 & -1.086 \\
\hline-7 & -0.02279 & -0.465 & $-0.06787^{* *}$ & -2.275 & 0.00117 & 0.103 & -0.03082 & -1.293 \\
\hline-5 & $-0.02308^{*}$ & -1.706 & $-0.09573^{* *}$ & -2.430 & 0.00328 & -0.207 & -0.04090 & -1.241 \\
\hline-4 & 0.01043 & 0.362 & $-0.08529 * *$ & -2.017 & 0.01953 & 1.189 & -0.02136 & -0.517 \\
\hline-3 & $-0.04414^{* *}$ & -2.327 & $-0.12944^{* *}$ & -2.482 & $-0.03358 *$ & -1.655 & -0.05494 & -1.603 \\
\hline-2 & 0.00352 & 0.362 & $-0.12592^{* *}$ & -2.223 & -0.00390 & -0.052 & -0.05885 & -1.551 \\
\hline-1 & -0.02175 & -1.293 & $-0.14767^{* *}$ & -2.223 & -0.02708 & -1.189 & -0.08593 & -1.551 \\
\hline 3 & -0.01503 & -0.931 & $-0.26363^{* * *}$ & -2.999 & -0.00602 & -0.621 & $-0.20229 * * *$ & -2.637 \\
\hline 4 & 0.00564 & 0.207 & $-0.25799^{* * *}$ & -3.051 & 0.00931 & 0.621 & $-0.19298^{* *}$ & -2.327 \\
\hline 5 & -0.00169 & -0.362 & $-0.25968^{* * *}$ & -3.361 & 0.00389 & 0.362 & $-0.18908^{* *}$ & -2.379 \\
\hline 6 & -0.00961 & -0.414 & $-0.26930^{* * *}$ & -3.258 & 0.00096 & 0.465 & $-0.18812^{* *}$ & -2.275 \\
\hline 7 & 0.02406 & 1.241 & $-0.24523^{* * *}$ & -2.896 & 0.02362 & 1.241 & -0.16450 & -1.551 \\
\hline 8 & -0.01877 & -1.603 & $-0.26400^{* * *}$ & -3.309 & -0.01651 & -1.293 & $-0.18101^{* *}$ & -2.120 \\
\hline 9 & -0.01527 & -0.414 & $-0.27928^{* * *}$ & -3.258 & -0.01599 & -0.310 & $-0.19701^{*}$ & -1.913 \\
\hline 10 & -0.00036 & -0.776 & $-0.27964^{* * *}$ & -3.464 & -0.01011 & -1.500 & $-0.20713^{* *}$ & -2.430 \\
\hline \multicolumn{9}{|c|}{ ARs and CARs following surprise neutral rating news during crisis period } \\
\hline-6 & -0.00003 & -0.004 & 0.01010 & 0.592 & -0.00046 & -0.058 & 0.00632 & 0.418 \\
\hline-5 & 0.05121 & 1.172 & 0.06132 & 1.464 & 0.04977 & 1.138 & 0.05609 & 1.318 \\
\hline-4 & -0.01506 & -1.206 & 0.04625 & 1.33 & -0.01594 & -1.393 & 0.04014 & 1.107 \\
\hline-3 & -0.00499 & -0.563 & 0.04126 & 1.352 & -0.00235 & -0.287 & 0.03779 & 1.177 \\
\hline-2 & $0.02030 * *$ & 2.515 & $0.06156^{* *}$ & 2.183 & $0.02045^{* * *}$ & 2.732 & $0.05824^{*}$ & 1.991 \\
\hline-1 & 0.01183 & 1.057 & $0.07340^{* *}$ & 2.344 & 0.01040 & 0.872 & $0.06865^{* *}$ & 2.074 \\
\hline 0 & 0.00148 & 0.154 & $0.07488^{* *}$ & 2.333 & -0.00361 & -0.401 & $0.06504^{*}$ & 1.897 \\
\hline 1 & $-0.01864^{*}$ & -1.903 & $0.05624^{*}$ & 1.701 & $-0.01935^{* *}$ & -2.146 & 0.04569 & 1.319 \\
\hline 2 & $-0.02720^{* *}$ & -2.377 & 0.02904 & 0.988 & $-0.03013^{* * *}$ & -2.832 & 0.01555 & 0.481 \\
\hline 3 & $0.01557^{*}$ & 2.010 & 0.04461 & 1.430 & $0.01552^{*}$ & 1.831 & 0.03108 & 0.917 \\
\hline 4 & 0.00476 & 0.518 & 0.04937 & 1.739 & 0.00414 & 0.448 & 0.03522 & 1.101 \\
\hline 5 & 0.00043 & 0.040 & $0.04981^{*}$ & 1.914 & -0.00322 & -0.308 & 0.03199 & 1.136 \\
\hline 6 & -0.00894 & -1.207 & 0.04087 & 1.567 & -0.00644 & -0.926 & 0.02555 & 0.893 \\
\hline 7 & -0.00845 & -0.592 & 0.03241 & 1.065 & -0.01043 & -0.746 & 0.01512 & 0.459 \\
\hline 8 & -0.00364 & -0.499 & 0.02877 & 0.886 & -0.00507 & -0.726 & 0.01004 & 0.295 \\
\hline 9 & -0.01235 & -1.381 & 0.01641 & 0.504 & $-0.01518^{*}$ & -1.757 & -0.00513 & -0.143 \\
\hline 10 & -0.00084 & -0.094 & 0.01557 & 0.468 & 0.00064 & 0.079 & -0.00449 & -0.117 \\
\hline
\end{tabular}

$* * *, * *$ and $*$ denote respectively significance at $1 \%, 5 \%$ and $10 \%$ levels 


\section{Macrothink \\ International Journal of Accounting and Financial Reporting \\ ISSN 2162-3082 \\ 2018, Vol. 8, No. 3}

ARs and CARs following expected neutral rating news during non-crisis period

\begin{tabular}{|c|c|c|c|c|c|c|c|c|}
\hline \multicolumn{3}{|c|}{ Stock adjusted returns } & \multicolumn{6}{|c|}{ OLS Market model returns } \\
\hline Day (t) & t statis & & CAR & t statistic & AR & t statistic & CAR & t statistic \\
\hline-10 & $-0.01061^{*}$ & -1.867 & $-0.01061^{*}$ & -1.867 & $-0.01044^{* *}$ & -2.127 & $-0.01044^{* *}$ & -2.127 \\
\hline-9 & -0.00011 & 0.666 & -0.01073 & -0.925 & -0.00032 & 0.568 & -0.01076 & -0.990 \\
\hline-8 & $-0.00464^{* * *}$ & -2.646 & $-0.01537^{*}$ & -1.802 & $-0.00415^{* *}$ & -2.289 & -0.01491 & -1.640 \\
\hline-7 & -0.00150 & -0.795 & $-0.01688^{* * *}$ & -2.613 & -0.00014 & -0.925 & $-0.01505^{* *}$ & -2.321 \\
\hline-6 & -0.00009 & -0.081 & $-0.01698^{* *}$ & -2.289 & -0.00074 & -0.308 & $-0.01579^{*}$ & -1.769 \\
\hline-5 & -0.00327 & -0.179 & $-0.02025^{*}$ & -1.932 & -0.00337 & -0.081 & $-0.01916^{*}$ & -1.834 \\
\hline-4 & -0.00145 & -0.211 & $-0.02170^{* *}$ & -2.127 & -0.00084 & 0.114 & -0.02001 & -1.640 \\
\hline-3 & -0.00327 & -1.542 & $-0.02497^{*}$ & -1.672 & -0.00321 & -1.477 & -0.02322 & -1.380 \\
\hline-2 & -0.00814 & -0.016 & -0.03312 & -1.477 & -0.00896 & -0.179 & -0.03219 & -1.120 \\
\hline-1 & -0.00041 & 0.308 & -0.03353 & 1.510 & 0.00014 & 0.308 & -0.03204 & -1.380 \\
\hline 0 & -0.00069 & -0.503 & -0.03423 & -1.542 & -0.00040 & -0.698 & -0.03245 & -1.282 \\
\hline 1 & -0.00004 & 0.179 & -0.03427 & -1.510 & 0.00027 & 0.114 & -0.03217 & -1.120 \\
\hline 2 & -0.00032 & -0.049 & -0.03459 & -1.575 & 0.00062 & 0.958 & -0.03155 & -1.088 \\
\hline 3 & $-0.00787^{*}$ & -1.899 & $-0.04247^{* *}$ & -2.094 & -0.00634 & -1.445 & $-0.03789^{*}$ & -1.802 \\
\hline 4 & $0.00474^{*}$ & 1.769 & $-0.03772^{*}$ & -1.899 & $0.00453^{*}$ & 1.737 & -0.03335 & -1.347 \\
\hline 5 & -0.00203 & -1.088 & $-0.03975^{*}$ & -1.802 & -0.00096 & -0.406 & -0.03432 & -1.315 \\
\hline 6 & -0.00097 & -0.568 & $-0.04073^{*}$ & -1.899 & 0.00082 & 0.438 & -0.03350 & -1.217 \\
\hline 7 & -0.00113 & -0.146 & $-0.04187^{*}$ & -1.802 & -0.00097 & -0.114 & -0.03447 & -1.282 \\
\hline 8 & 0.00021 & -0.081 & $-0.04166^{*}$ & -1.802 & -0.00023 & -0.146 & -0.03471 & -1.185 \\
\hline 9 & 0.00001 & -0.081 & -0.04165 & -1.542 & 0.00178 & 0.601 & -0.03292 & -1.023 \\
\hline 10 & -0.00133 & -0.373 & -0.04298 & -1.510 & -0.00114 & -0.146 & -0.03406 & -1.088 \\
\hline \multicolumn{9}{|c|}{ ARs and CARs following surprise neutral rating news during non-crisis period } \\
\hline \multicolumn{5}{|c|}{ Stock adjusted returns } & \multicolumn{4}{|c|}{ OLS Market model returns } \\
\hline Day (t) & AR & t statistic & CAR & t statistic & AR & t statistic & CAR & t statistic \\
\hline-10 & 0,00121 & 0.270 & 0.00121 & 0.476 & 0,00036 & -0.050 & 0.00036 & 0.155 \\
\hline-9 & 0,00146 & 0.687 & 0.00267 & 0.764 & 0,00077 & 0.529 & 0.00114 & 0.350 \\
\hline-8 & $-0,00113$ & -0.420 & 0.00153 & 0.359 & $-0,00263$ & -1.105 & -0.00148 & -0.355 \\
\hline-7 & 0,00313 & 2.295 & 0.00467 & 1.140 & 0,00239 & 1.864 & 0.00091 & 0.224 \\
\hline-6 & 0,00104 & 0.554 & 0.00571 & 1.713 & 0,00120 & 0.589 & 0.00211 & 0.449 \\
\hline-5 & $-0,00245$ & -1.166 & 0.00325 & 0.578 & $-0,00284$ & -1.441 & -0.00072 & -0.133 \\
\hline-4 & $-0,00142$ & -0.338 & 0.00183 & 0.294 & $-0,00177$ & -0.381 & -0.00250 & -0.423 \\
\hline-3 & 0,00644 & 1.978 & 0.00827 & 1.087 & 0,00602 & 1.659 & 0.00351 & 0.483 \\
\hline-2 & $-0,00221$ & -0.546 & 0.00605 & 0.777 & $-0,00284$ & -0.707 & 0.00067 & 0.083 \\
\hline-1 & 0,00272 & 0.766 & 0.00877 & 0.962 & 0,00290 & 0.820 & 0.00358 & 0.367 \\
\hline 0 & $-0,00873$ & -2.296 & 0.00004 & 0.042 & $-0,00897$ & -2.481 & -0.00539 & -0.544 \\
\hline 1 & $-0,00026$ & -0.135 & -0.00022 & -0.025 & $-0,00274$ & -0.910 & -0.00813 & -0.875 \\
\hline 2 & $-0,00121$ & -0.023 & -0.00143 & -0.157 & $-0,00129$ & -0.138 & -0.00943 & -0.964 \\
\hline 3 & $-0,00271$ & -1.223 & -0.00415 & -0.452 & $-0,00307$ & -1.422 & -0.01250 & -1.246 \\
\hline 4 & $-0,00012$ & -0.284 & -0.00428 & -0.485 & $-0,00220$ & -1.403 & -0.01471 & -1.524 \\
\hline 5 & 0,00050 & 0.482 & -0.00378 & -0.434 & $-0,00065$ & -0.099 & -0.01536 & -1.628 \\
\hline 6 & $-0,00139$ & -0.582 & -0.00517 & -0.606 & $-0,00203$ & -0.783 & $-0.01739^{*}$ & -1.846 \\
\hline 7 & 0,00106 & 0.567 & -0.00411 & -0.447 & 0,00090 & 0.515 & -0.01649 & -1.662 \\
\hline 8 & $-0,00210$ & -0.927 & -0.00621 & -0.665 & $-0,00226$ & -0.953 & $-0.01876^{*}$ & -1.901 \\
\hline 9 & $-0,00096$ & -0.197 & -0.00717 & -0.787 & $-0,00089$ & -0.219 & $-0.01965^{*}$ & -1.993 \\
\hline 10 & $-0,00091$ & -0.387 & -0.00809 & -0.879 & $-0,00133$ & -1.234 & $-0.02099 * *$ & -2.094 \\
\hline
\end{tabular}

\section{Copyright Disclaimer}

Copyright for this article is retained by the author(s), with first publication rights granted to the journal.

This is an open-access article distributed under the terms and conditions of the Creative Commons Attribution license (http://creativecommons.org/licenses/by/4.0/) 